INTERNATIONAL JOURNAL OF MODERN PHYSICS B

(CONDENSED MATTER PHYSICS; STATISTICAL PHYSICS; ATOMIC, MOLECULAR AND OPTICAL PHYSICS)

ISSN (print): 0217-9792 | ISSN (online): 1793-6578

PUBLISHER: WORLD SCIENTIFIC (SINGAPORE)

Accepted: January $4^{\text {th }} 2019$

\title{
UNSTEADY FREE CONVECTIVE HEAT TRANSFER IN THIRD-GRADE FLUID FLOW FROM AN ISOTHERMAL VERTICAL PLATE: A THERMODYNAMIC ANALYSIS
}

\author{
Ashwini Hiremath ${ }^{1}$, G. Janardhana Reddy ${ }^{1,}$, Mahesh Kumar ${ }^{1}$ and O. Anwar Bég ${ }^{2}$ \\ ${ }^{1}$ Department of Mathematics, Central University of Karnataka, Kalaburagi, India \\ ${ }^{2}$ Fluid Mechanics, Aeronautical and Mechanical Engineering Department, School of Computing, \\ Science and Engineering, University of Salford, Manchester M54WT, UK \\ *Corresponding author-Email: gjr@cuk.ac.in (extra corrections in green)
}

\begin{abstract}
:
The current study investigates theoretically and numerically the entropy generation in timedependent free-convective third-grade viscoelastic fluid convection flow from a vertical plate. The non-dimensional conservation equations for mass, momentum, and energy are solved using a Crank-Nicolson finite difference method with suitable boundary conditions. Expressions for known values of flow-variables coefficients are also derived for the wall heat transfer and skin friction and numerically evaluated. The effect of Grashof number, Prandtl number, group parameter (product of dimensionless temperature difference and Brinkman number) and thirdgrade parameter on entropy heat generation is analyzed and shown graphically. Bejan line distributions are also presented for the influence of several control parameters. The computations reveal that with increasing third-grade parameter the entropy generation decreases and Bejan number increases. Also, the comparison graph shows that contour lines for third-grade fluid vary considerably from the Newtonian fluid. The study is relevant to non-Newtonian thermal materials processing systems.
\end{abstract}

KEYWORDS: Free convection; Vertical plate; Third-grade non-Newtonian fluid, Finite difference method, Entropy heat generation, Bejan lines, Bejan number.

\section{NOMENCLATURE}

\section{Roman}

$C_{p} \quad$ specific heat at constant pressure 
Be dimensionless Bejan number

$\bar{C}_{f} \quad$ dimensionless average momentum transport coefficient

$\mathrm{g}^{\prime} \quad$ acceleration due to gravity

$k \quad$ thermal conductivity

$G r \quad$ Grashof number

$\overline{\mathrm{Nu}} \quad$ average heat transport coefficient

$\mathrm{Br} \quad$ Brinkman number

$l \quad$ dimensional length of the plate

$t^{\prime} \quad$ time

$P \quad$ fluid pressure

I identity tensor

$\operatorname{Pr} \quad$ Prandtl number

$T^{\prime} \quad$ temperature

$t \quad$ dimensionless time

$T \quad$ dimensionless temperature

Ns dimensionless entropy heat generation number

$T^{* *} \quad$ matrix transposition

$\frac{d}{d t} \quad$ material time derivative

$S_{1}^{*}, S_{2}^{*}, S_{3}^{*}$ Rivlin and Ericksen tensors

tr matrix trace

$\bar{x} \quad$ axial coordinate

X dimensionless axial coordinate

$\bar{y} \quad$ transverse coordinate

$Y \quad$ dimensionless transverse coordinate

$\bar{u}, \bar{v} \quad$ components of velocity vector in $(\bar{x}, \bar{y})$ coordinate system 
$U, V$ dimensionless components of velocity vector in $X, Y$ directions, respectively

\section{Greek letters}

$\alpha_{1}{ }^{*}, \alpha_{2}{ }^{*}, \beta_{1}{ }^{*}, \beta_{2}{ }^{*}, \beta_{3}{ }^{*} \quad$ rheological material moduli

$\alpha_{1} \quad$ viscoelastic parameter

$\alpha_{2} \quad$ second-grade fluid parameter

$\alpha_{3} \quad$ third-grade fluid parameter

$\beta_{T} \quad$ volumetric coefficient of thermal expansion

$\tau^{*} \quad$ Cauchy stress tensor

$\rho \quad$ density of third-grade fluid

$\alpha \quad$ thermal diffusivity

$\Omega \quad$ non-dimensional temperature difference

$\mu \quad$ viscosity of third-grade fluid

$B r \Omega^{-1}$ non-dimensional group parameter

$\vartheta \quad$ kinematic viscosity of third-grade fluid

\section{Subscripts}

$w \quad$ wall conditions

$\infty \quad$ ambient conditions

$f, g$ grid levels in $(X, Y)$ coordinate system

\section{Superscript}

$h \quad$ time level

\section{INTRODUCTION}


Significant mathematical (and experimental) investigation of thermal convection boundary layer flows was greatly assisted by Prandtl's [1] boundary layer theory which reduces the complexity of the governing equations considerably. The inclusion of viscous effects significantly advanced earlier limitations of the potential flow theory. Using Prandtl's boundary layer theory, Blasius [2] examined the two-dimensional flow on a flat surface. Over a century after Prandtl's monumental work, studies on boundary layer theory have remained a major area for researchers. Boundary layers arise in a tremendous range of industrial and natural fluid phenomena including atmospheric flows, geothermal plumes, paper production, metallic sheet synthesis, polymer dynamics, drag force computation for aero/hydronautical bodies, mixing techniques, aerodynamics (reattachment and separation), coating flows, biofluid dynamics etc. Thermal convection boundary layers may involve free, mixed or forced convection. Accurate simulation of the heat transfer in these flows is a critical area of interest to engineers and scientists.

Thermal boundary layer flows driven by buoyancy forces external to different geometries are of some interest in the cooling of electronic devices, environmental comfort, cores of a nuclear reactor, thermal storage, grain drying, geophysics, coating dynamics and many others. Many studies have been communicated considered such flows adjacent to both flat surfaces (vertical, inclined plates) and curved bodies (cones, cylinders, curved walls etc). Out of these, the vertical flat plate is a geometry of considerable interest in many areas of technology including solar collectors, fire dynamics (combustion), coating in materials processing etc. Vertical plate free convection has been examined for an extensive range of multi-physical problems. Merkin [3] presented asymptotic solutions for general similarity free convection flows over a vertical plate considering the case of constant heat flux. Mansour [4] considered radiative heat transfer effects in oscillatory free-convective flow from a vertical plate with the Rosseland approximation. Takhar et al. [5] employed a series expansion method for free-convective flow in the presence of a magnetic field, radiative flux and thermal buoyancy. Kuznetsov and Nield [6] derived a similarity solution for nanofluid flow over a vertical plate and showed the dependency of the reduced Nusselt 
number on thermophoresis, Brownian motion \& buoyancy-ratio parameters. Kuznetsov and Nield [7] further revised the nanofluid free convection boundary layer model in [6] to give a more realistic solution where the particle fraction of nanofluid is controlled passively on the boundary. Very recently Biswas et al. [8] used the explicit finite difference to compute solutions for the flow past a plate. Nevertheless, all these studies are confined to Newtonian fluids. The Newtonian classical fluid theory cannot simulate many sophisticated phenomena exhibited by the real industrial fluids (slurries, plastics, coating materials, gels etc) such as non-linear creep, stress relaxation, normal stress differences involved in shear flows, threshold stress developed at the onset of fluid flow, shear thickening/thinning and viscosity dependent stress. However various non-Newtonian models (such as Casson fluid model [9], [10] and couple stress fluid model [11]) are available which can explain these and other phenomena satisfactorily. These models include thixotropic models, viscoelastic models, memory fluids, microstructural fluid models etc. The implicit relationship of deformation gradient and history of stress can be quite well simulated with the Reiner-Rivlin family of fluid models of the differential type. The third-grade fluid model is an advanced sub-set of the generalized differential type model. A key attraction to engineers of this model is that it has been shown to be capable of simulating accurately shear-thinning or thickening phenomena. Various lubricants and coolants, molten plastic and polymers employed in chemical industries, polymeric solutions, slurries, blood, and other physiological fluids are approximated reasonably well with this fluid model. Mathematical and computational studies of third-grade fluid dynamics have therefore received substantial interest in recent years. Ayub et al. [12] have presented exact solutions for third-grade fluid boundary layer flow from a porous vertical plate. Busuioc and Iftimie [13] have rigorously demonstrated the existence of global and unique solutions for equations of third-grade fluids. Akyildiz et al. [14] have compared exact and numerical solutions for third-grade fluid flow from a rotating cylindrical geometry. Sajid et al. [15] presented homotopy analytical solutions for hydromagnetic third-grade convection boundary layer flow from a linear stretching sheet for both cases of prescribed surface temperature (PST- 
case) and prescribed surface heat flux (PHF-case), observing that the flow is decelerated with increasing third-grade viscoelastic parameter. Bég et al. [16] presented finite element numerical solution for viscoelastic third-grade fluid convection in nonlinear porous media. Sahoo [17] presented finite difference computational solutions for hydromagnetic Hiemenz flow and thermal convection in a third-grade fluid confirming that the velocity gradient at the wall is suppressed with a greater third-grade viscoelastic parameter. Sahoo and Do [18] investigated the influence of partial slip from a magnetized heated third-grade fluid along a stretching sheet. They showed that increasing third-grade parameter amplifies momentum boundary layer thickness (i.e. induces flow deceleration) and diminishes thermal boundary layer thickness (i.e. causes cooling) and that these effects are the reverse to those generated by increasing slip. Sahoo and Poncet [19] analyzed the magnetohydrodynamic heat transfer in third-grade fluid flow. Keimanesh et al. [20] used the multistep DTM (differential transformation method) to study channel flow of a third-grade fluid with heat transfer. Baoku et al. [21] investigated suction/injection effects on third-grade convection flow with a numerical scheme using Richardson's extrapolation. Hayat et al. [22] derived series solutions for the radiative-convective flow of a third-grade fluid. Nadeem and Saleem [23] examined the viscoelastic third-grade nanofluid transport from a rotating cone. Adesanya and Makinde [24] studied heat source effects on the forced-convective flow of thirdgrade fluid using the Adomain decomposition method (ADM). Latif et al. [25] analyzed hydromagnetic third-grade fluid flow in a deformable symmetric channel considering the effects of viscous dissipation. Abbas et al. [26] presented numerical solutions for reactive species diffusion in a stagnation-point flow of a third-grade fluid. Hayat et al. [27] considered the magneto-convection flow of a third-grade elastic-viscous nanofluid from a stretching sheet. Reddy et al. [28] recently investigated the heat line visualization for time-dependent third-grade fluid convection boundary layer flow external to a vertical cylinder. Reddy et al. [29] further simulated transient heat transfer in third-grade fluid flow from an upright cylinder under supercritical conditions. 
In recent years, engineers have actively sought new robust methods to improve thermal efficiency and control of energy wastage. A key challenge has been the successful design of a thermodynamic system (e.g. engines of vehicles, refrigerators, air conditioners, and thermal power devices) to achieve enhanced thermal performance using all available energy without loss or by minimizing loss. Many studies of thermodynamic system performance enhancement have been communicated and include multiple physical effects (magnetic, chemical, mechanical, electromagnetic, etc.) and these find diverse applications in mechanical engineering, industrial and energy systems applications. The irreversibility in the system is the product of heat transfer and a quantification of the wastage of the available energy (exergy). Frictional effects, chemical reactions, a transformation of mass, applied magnetic field effects, viscous dissipation, Joule heating, system turbulence, and temperature gradients are the causes for energy loss in thermodynamics. These factors increase the disorder of the system and thereby produce entropy heat generation. In irreversible processes, the generated entropy and the reduction of exergy have a direct relationship. Hence, by measuring the entropy it is feasible to minimize the reduction of exergy and maximize the thermal performance of the system. The minimization of entropy generation is closely associated with the first and second law of thermodynamics and the system design. The first law of thermodynamics provides engineers with only a quantitative measurement of the system energy and has limited benefits. In contrary, the second law of thermodynamics reveals critical information relating to the qualitative part, i.e. "Every actual spontaneous process is irreversible". Henceforth, 'the entropy generation minimization' technique is equivalent to the 'second law of thermodynamics'. Entropy analysis has emerged in the past several decades as a major focus of interest in chemical reactors, combustion, turbo-machines, industrial thermal technologies, heat exchangers, cooling devices, electric conductors, hot water \& steam pipes, refrigerator, heater, gas turbines, glass blowing, continuous casting of metals, plastic production, extrusion of plastic sheets, polymers in metal spring processer, etc. Primarily, the idea about entropy was put forward in detailed experiments by Carnot [30] in 1824. However, the idea of 
minimizing entropy generation to elevate thermal efficiency was first proposed by the American engineer, Bejan [31-35]. Entropy generation minimization (EGM) studies have been extensive in recent years for a variety of fluids and physical phenomena present in industrial processes. Makinde and Bég [36] used the perturbation method with Hermite-Pade' approximations to compute Bejan numbers in EGM analysis of hydromagnetic channel flow with chemical reaction effects. Magherbi et al. [37] presented control volume finite-element method computations to evaluate the variations of the total entropy generation as a function of time for Rayleigh number in the natural-convective flow. Mahmud and Fraser [38] presented a rigorous analysis of entropy generation in a tube and parallel plate channel convection flows, computing both entropy generation number and Bejan number profiles. Entropy generation analysis due to natural convection has been investigated for different geometries including a square cavity [39], open triangular cavity [40] and vertical cylinder [41]. Also, some research works from an application point of view are listed in the reference section [42-45].

In the present work, thermodynamic optimization (entropy generation minimization) is conducted for unsteady free-convective third-grade fluid boundary layer flow from an isolated vertical flat plate by determining the losses caused due to viscous effects and heat transfer. The second law of thermodynamics has been applied for a present problem. An appropriate temperature difference is maintained to investigate the heat transfer effects. The mathematical model derived is normalized with appropriate transformations and the resulting nonlinear boundary value problem which comprises a set of coupled, highly non-linear equations is solved with an efficient and stable Crank-Nicholson finite difference technique. Extensive visualization of time-dependent velocity profiles $(U)$, time-dependent temperature profiles $(T)$, average momentum transport coefficient $\left(\overline{C_{f}}\right)$ profiles, average heat transport coefficient $(\overline{N u})$ profiles, transient and steadystate entropy generation distributions $(N s)$, transient and steady-state Bejan number profiles $(B e)$, unsteady state contours of temperature $(T) \&$ velocity $(U)$ are presented for the effects of Grashof number $(G r)$, group parameter (Brinkman number divided by temperature difference, $B r \Omega^{-1}$ ), 
Prandtl number $(P r)$ and third-grade non-Newtonian parameter $\left(\alpha_{3}\right)$. Validation of the with previous studies is included. Grid independence and time independence numerical tests are also conducted. The present work addresses theoretically and numerically the minimization of entropy generation in third-grade viscoelastic fluid (such as a polymer, slurry, lubricant, molten plastic, etc.) flows. The current simulations are therefore highly relevant to thermal materials processing and rheological coating dynamics. Also, radiation shields, mechanical supports, electric cables, counter flow heat exchangers are some of the industrial components designed to minimize heat loss and pertinent application of entropy generation minimization analysis.

\section{PROBLEM FORMULATION}

Thermal convection boundary layer flow in a non-Newtonian third-grade fluid along a vertical flat plate is investigated. The heated semi-infinite vertical plate is kept stationary comparative to the flow of fluid and the physical system is illustrated in Fig.1. A two-dimensional rectangular coordinate system is selected where plate (along the $\bar{x}$-axis) is placed normal to the flow direction (along $\bar{y}$-axis). The third-grade fluid within which the plate is immersed is maintained at $T_{\infty}^{\prime}$. At $t^{\prime}=0$, the plate is also sustained at the same temperature. For an increased time $\left(t^{\prime}>0\right)$, a temperature difference is maintained such that the plate temperature is increased to $T_{w}^{\prime}$, and this is maintained at all later times.

\subsection{The constitutive equation:}

The Cauchy stress-tensor $\left(\tau^{*}\right)$ for a third-grade fluid having thermodynamic compatibility (given by Fosdick and Rajagopal [46]) is as follows:

$$
\tau^{*}=-P I+\mu S_{1}^{*}+\alpha_{1}^{*} S_{2}^{*}+\alpha_{2}^{*} S_{1}^{* 2}+\beta_{1}^{*} S_{3}^{*}+\beta_{2}^{*}\left(S_{1}^{*} S_{2}^{*}+S_{2}^{*} S_{1}^{*}\right)+\beta_{3}^{*}\left(\operatorname{tr} S_{1}^{* 2}\right) S_{1}^{*}
$$


Here the spherical part of stress relation is specified by a factor $-P I$, the temperature dependent material moduli are $\beta_{j}^{*}(j=1,2,3)$ and $\alpha_{j}^{*}(j=1,2)$, and the Rivlin-Ericksen tensor matrices $S_{J}^{*}(j=$ $1,2,3)$ are given by:

$S_{1}^{*}=(\nabla \boldsymbol{V})^{T^{* *}}+\nabla \boldsymbol{V}$

$S_{J}^{*}=\frac{d S_{j-1}^{*}}{d t}+(\nabla \boldsymbol{V})^{T^{* *}} S_{J-1}^{*}+S_{J-1}^{*}(\nabla \boldsymbol{V}), \quad j=2,3 \ldots$

where $T^{* *}$ is transposition of matrix, $\boldsymbol{V}$ is velocity vector, $\nabla$ is the gradient operator, and $\frac{d}{d t}$ i.e. material derivative is expressed as:

$$
\frac{d}{d t}(\cdot)=\left(\frac{\partial}{\partial t}+V \nabla\right)(\cdot)
$$

Also, two more assumptions for the fluid motion are listed, i.e., (i) Clausius-Duhem inequality, (ii) least value of Helmholtz free energy at equilibrium. The constraints for this fluid model are defined as,

$$
\begin{aligned}
& \mu \geq 0 ; \quad \alpha_{1}^{*} \geq 0 ; \quad\left|\alpha_{1}^{*}+\alpha_{2}^{*}\right| \leq \sqrt{24 \mu \beta_{3}^{*}} \\
& \left.\beta_{1}^{*}=0 ; \quad \beta_{2}^{*}=0 ; \quad \beta_{3}^{*} \geq 0\right\}
\end{aligned}
$$

These conditions on the material moduli are discussed in [47], [48] and are substituted in Eq. (1) reduces to:

$$
\tau^{*}=-P I+\mu S_{1}^{*}+\alpha_{1}^{*} S_{2}^{*}+\alpha_{2}^{*} S_{1}^{* 2}+\beta_{3}^{*}\left(\operatorname{tr} S_{1}^{* 2}\right) S_{1}^{*}
$$

Incorporating the above non-Newtonian third-grade model conditions, the mass and momentum conservation boundary layer equations for the flow of third-grade fluid are given by [28, 29]:

$$
\begin{aligned}
& \frac{\partial \bar{u}}{\partial \bar{x}}+\frac{\partial \bar{v}}{\partial \bar{y}}=0 \\
& \rho \frac{D \bar{u}}{D t}=-\frac{\partial P}{\partial \bar{x}}+\frac{\partial \tau_{\bar{x}}^{*}}{\partial \bar{x}}+\frac{\partial \tau_{\overline{x y}}^{*}}{\partial \bar{y}}
\end{aligned}
$$


$\rho \frac{D \bar{v}}{D t}=-\frac{\partial P}{\partial \bar{y}}+\frac{\partial \tau_{\overline{y x}}^{*}}{\partial \bar{x}}+\frac{\partial \tau_{\overline{y y}}^{*}}{\partial \bar{y}}$

Here the following definitions apply:

$$
\begin{aligned}
& \tau_{\bar{x} \bar{y}}^{*}=\tau_{\bar{y} \bar{x}}^{*}=\mu\left(\frac{\partial \bar{u}}{\partial \bar{y}}+\frac{\partial \bar{v}}{\partial \bar{x}}\right)+\alpha_{1}^{*}\left[\frac{\partial^{2} \bar{u}}{\partial t^{\prime} \partial \bar{y}}+\frac{\partial^{2} \bar{v}}{\partial t^{\prime} \partial \bar{x}}+\bar{u} \frac{\partial^{2} \bar{u}}{\partial \bar{x} \partial \bar{y}}+\bar{u} \frac{\partial^{2} \bar{v}}{\partial \bar{x}^{2}}+\bar{v} \frac{\partial^{2} \bar{u}}{\partial \bar{y}^{2}}+\bar{v} \frac{\partial^{2} \bar{v}}{\partial \bar{y} \partial \bar{x}}+3 \frac{\partial \bar{u}}{\partial \bar{x}} \frac{\partial \bar{u}}{\partial \bar{y}}+\right. \\
& \left.\frac{\partial \bar{w}}{\partial \bar{x}} \frac{\partial \bar{w}}{\partial \bar{y}}+3 \frac{\partial \bar{v}}{\partial \bar{x}} \frac{\partial \bar{v}}{\partial \bar{y}}+\frac{\partial \bar{u}}{\partial \bar{y}} \frac{\partial \bar{v}}{\partial \bar{y}}+\frac{\partial \bar{u}}{\partial \bar{z}} \frac{\partial \bar{w}}{\partial \bar{y}}+\frac{\partial \bar{u}}{\partial \bar{x}} \frac{\partial \bar{v}}{\partial \bar{x}}+\frac{\partial \bar{v}}{\partial \bar{z}} \frac{\partial \bar{w}}{\partial \bar{x}}\right]+\alpha_{2}^{*}\left[2 \frac{\partial \bar{u}}{\partial \bar{x}} \frac{\partial \bar{u}}{\partial \bar{y}}+2 \frac{\partial \bar{v}}{\partial \bar{x}} \frac{\partial \bar{u}}{\partial \bar{x}}+\right. \\
& \left.2 \frac{\partial \bar{u}}{\partial \bar{y}} \frac{\partial \bar{v}}{\partial \bar{y}}+2 \frac{\partial \bar{v}}{\partial \bar{y}} \frac{\partial \bar{v}}{\partial \bar{x}}+\frac{\partial \bar{w}}{\partial \bar{x}} \frac{\partial \bar{v}}{\partial \bar{z}}+\frac{\partial \bar{u}}{\partial \bar{z}} \frac{\partial \bar{w}}{\partial \bar{y}}+\frac{\partial \bar{w}}{\partial \bar{x}} \frac{\partial \bar{w}}{\partial \bar{y}}+\frac{\partial \bar{u}}{\partial \bar{z}} \frac{\partial \bar{v}}{\partial \bar{z}}\right]+4 \beta_{3}^{*}\left[\left(\frac{\partial \bar{u}}{\partial \bar{x}}\right)^{2}+\left(\frac{\partial \bar{v}}{\partial \bar{y}}\right)^{2}+\right. \\
& \left.\left(\frac{\partial \bar{w}}{\partial \bar{z}}\right)^{2}+\frac{1}{2}\left\{\left(\frac{\partial \bar{v}}{\partial \bar{x}}+\frac{\partial \bar{u}}{\partial \bar{y}}\right)^{2}+\left(\frac{\partial \bar{w}}{\partial \bar{x}}+\frac{\partial \bar{u}}{\partial \bar{z}}\right)^{2}+\left(\frac{\partial \bar{w}}{\partial \bar{y}}+\frac{\partial \bar{v}}{\partial \bar{z}}\right)^{2}\right\}\right]\left(\frac{\partial \bar{v}}{\partial \bar{x}}+\frac{\partial \bar{u}}{\partial \bar{y}}\right) \\
& \tau_{\bar{x} \bar{x}}^{*}=2 \mu\left(\frac{\partial \bar{u}}{\partial \bar{x}}\right)+\alpha_{1}^{*}\left\{2 \frac{\partial^{2} \bar{u}}{\partial t^{\prime} \partial \bar{x}}+2 \bar{u} \frac{\partial^{2} \bar{u}}{\partial \bar{x}^{2}}+2 \bar{v} \frac{\partial^{2} \bar{u}}{\partial \bar{y} \partial \bar{x}}+2\left[2\left(\frac{\partial \bar{u}}{\partial \bar{x}}\right)^{2}+\frac{\partial \bar{v}}{\partial \bar{x}}\left(\frac{\partial \bar{u}}{\partial \bar{y}}+\frac{\partial \bar{v}}{\partial \bar{x}}\right)+\frac{\partial \bar{w}}{\partial \bar{x}}\left(\frac{\partial \bar{u}}{\partial \bar{z}}+\right.\right.\right. \\
& \left.\left.\left.\frac{\partial \bar{w}}{\partial \bar{x}}\right)\right]\right\}+\alpha_{2}^{*}\left\{\left(\frac{\partial \bar{v}}{\partial \bar{x}}+\frac{\partial \bar{u}}{\partial \bar{y}}\right)^{2}+4\left(\frac{\partial \bar{u}}{\partial \bar{x}}\right)^{2}+\left(\frac{\partial \bar{u}}{\partial \bar{z}}+\frac{\partial \bar{w}}{\partial \bar{x}}\right)^{2}\right\}+8 \beta_{3}^{*}\left[\left(\frac{\partial \bar{u}}{\partial \bar{x}}\right)^{2}+\left(\frac{\partial \bar{v}}{\partial \bar{y}}\right)^{2}+\right. \\
& \left.\left(\frac{\partial \bar{w}}{\partial \bar{z}}\right)^{2}+\frac{1}{2}\left\{\left(\frac{\partial \bar{u}}{\partial \bar{z}}+\frac{\partial \bar{w}}{\partial \bar{x}}\right)^{2}+\left(\frac{\partial \bar{v}}{\partial \bar{x}}+\frac{\partial \bar{u}}{\partial \bar{y}}\right)^{2}+\left(\frac{\partial \bar{w}}{\partial \bar{y}}+\frac{\partial \bar{v}}{\partial \bar{z}}\right)^{2}\right\}\right]\left(\frac{\partial \bar{u}}{\partial \bar{x}}\right) \\
& \tau_{\bar{y} \bar{y}}^{*}=2 \mu\left(\frac{\partial \bar{v}}{\partial \bar{y}}\right)+\alpha_{1}^{*}\left\{2 \frac{\partial^{2} \bar{v}}{\partial t^{\prime} \partial \bar{y}}+2 \bar{u} \frac{\partial^{2} \bar{v}}{\partial \bar{x} \partial \bar{y}}+2 \bar{v} \frac{\partial^{2} \bar{v}}{\partial \bar{y}^{2}}+2\left[2\left(\frac{\partial \bar{v}}{\partial \bar{y}}\right)^{2}+\frac{\partial \bar{u}}{\partial \bar{y}}\left(\frac{\partial \bar{u}}{\partial \bar{y}}+\frac{\partial \bar{v}}{\partial \bar{x}}\right)+\frac{\partial \bar{w}}{\partial \bar{y}}\left(\frac{\partial \bar{v}}{\partial \bar{z}}+\right.\right.\right. \\
& \left.\left.\left.\frac{\partial \bar{w}}{\partial \bar{y}}\right)\right]\right\}+\alpha_{2}^{*}\left\{\left(\frac{\partial \bar{v}}{\partial \bar{x}}+\frac{\partial \bar{u}}{\partial \bar{y}}\right)^{2}+4\left(\frac{\partial \bar{v}}{\partial \bar{y}}\right)^{2}+\left(\frac{\partial \bar{v}}{\partial \bar{z}}+\frac{\partial \bar{w}}{\partial \bar{y}}\right)^{2}\right\}+8 \beta_{3}^{*}\left[\left(\frac{\partial \bar{v}}{\partial \bar{y}}\right)^{2}+\left(\frac{\partial \bar{u}}{\partial \bar{x}}\right)^{2}+\right. \\
& \left.\left(\frac{\partial \bar{w}}{\partial \bar{z}}\right)^{2}+\frac{1}{2}\left\{\left(\frac{\partial \bar{u}}{\partial \bar{y}}+\frac{\partial \bar{v}}{\partial \bar{x}}\right)^{2}+\left(\frac{\partial \bar{v}}{\partial \bar{z}}+\frac{\partial \bar{w}}{\partial \bar{y}}\right)^{2}+\left(\frac{\partial \bar{u}}{\partial \bar{z}}+\frac{\partial \bar{w}}{\partial \bar{x}}\right)^{2}\right\}\right]\left(\frac{\partial \bar{v}}{\partial \bar{y}}\right)
\end{aligned}
$$

Here $(\bar{u}, \bar{v})$ are velocity components in the coordinate directions $(\bar{x}, \bar{y})$. The components of extra stress-tensor are denoted by $\tau_{\bar{y} \bar{y}}^{*}, \tau_{\bar{y} \bar{x}}^{*}, \tau_{\bar{x} \bar{y}}^{*}, \tau_{\bar{x} \bar{x}}^{*}$. Vertical fluid-flow is assumed by ignoring the transverse flow. Using the appropriate stress-tensors, the momentum equation is derived and along 
with energy equation define the thermal convection boundary layer flow equations for vertical plate free convection of a third-grade fluid under Boussinesq's approximation as:

$$
\begin{aligned}
& \frac{\partial \bar{u}}{\partial t^{\prime}}+\bar{u} \frac{\partial \bar{u}}{\partial \bar{x}}+\bar{v} \frac{\partial \bar{u}}{\partial \bar{y}}=\mathrm{g}^{\prime} \beta_{T}\left(T^{\prime}-T_{\infty}^{\prime}\right)+\vartheta \frac{\partial^{2} \bar{u}}{\partial \bar{y}^{2}}+\frac{\alpha_{1}^{*}}{\rho}\left[\frac{\partial^{3} \bar{u}}{\partial t^{\prime} \partial \bar{y}^{2}}+\frac{\partial \bar{u}}{\partial \bar{x}} \frac{\partial^{2} \bar{u}}{\partial \bar{y}^{2}}+\bar{u} \frac{\partial^{3} \bar{u}}{\partial \bar{x} \partial \bar{y}^{2}}+\bar{v} \frac{\partial^{3} \bar{u}}{\partial \bar{y}^{3}}+3 \frac{\partial^{2} \bar{u}}{\partial \bar{x} \partial \bar{y}} \frac{\partial \bar{u}}{\partial \bar{y}}\right] \\
&+\frac{\alpha_{2}^{*}}{\rho}\left[2 \frac{\partial \bar{u}}{\partial \bar{y}} \frac{\partial^{2} \bar{u}}{\partial \bar{x} \partial \bar{y}}\right]+\frac{\beta_{3}^{*}}{\rho}\left[6\left(\frac{\partial \bar{u}}{\partial \bar{y}}\right)^{2} \frac{\partial^{2} \bar{u}}{\partial \bar{y}^{2}}\right] \\
& \bar{u} \frac{\partial T^{\prime}}{\partial \bar{x}}+\bar{v} \frac{\partial T^{\prime}}{\partial \bar{y}}+\frac{\partial T^{\prime}}{\partial t^{\prime}}=\alpha \frac{\partial^{2} T^{\prime}}{\partial \bar{y}^{2}}
\end{aligned}
$$

The applied initial and boundary conditions:

$$
\begin{array}{lll}
t^{\prime} \leq 0: & T^{\prime}=T_{\infty}^{\prime}, \quad \bar{v}=0, \quad \bar{u}=0 & \text { for all } \bar{x} \text { and } \bar{y} \\
t^{\prime}>0: & T^{\prime}=T_{w}^{\prime}, \quad \bar{v}=0, \quad \bar{u}=0 & \text { at } \bar{y}=0 \\
& T^{\prime}=T_{\infty}^{\prime}, \quad \bar{v}=0, \quad \bar{u}=0 & \text { at } \bar{x}=0 \\
& T^{\prime} \rightarrow T_{\infty}^{\prime}, \quad \bar{v} \rightarrow 0, \quad \bar{u} \rightarrow 0, \frac{\partial \bar{u}}{\partial \bar{y}} \rightarrow 0 & \text { at } \bar{y} \rightarrow \infty
\end{array}
$$

The following dimensionless quantities are invoked:

$$
\begin{aligned}
& X=\frac{\bar{x} G r^{-1}}{l}, Y=\frac{\bar{y}}{l}, U=\left(\frac{\bar{u} l}{\vartheta}\right) G r^{-1}, \quad V=\frac{\bar{v} l}{\vartheta} \\
& t=\frac{\vartheta t^{\prime}}{l^{2}}, \quad G r=\frac{\mathrm{g}^{\prime} \beta_{T} l^{3}\left(T_{w}^{\prime}-T_{\infty}^{\prime}\right)}{\vartheta^{2}}, \quad T=\frac{T^{\prime}-T_{\infty}^{\prime}}{T_{w}^{\prime}-T_{\infty}^{\prime}}, \quad \operatorname{Pr}=\frac{\vartheta}{\alpha} \\
& \alpha_{1}=\frac{\alpha_{1}^{*}}{\rho l^{2}}, \quad \alpha_{2}=\frac{\alpha_{2}^{*}}{\rho l^{2}}, \quad \alpha_{3}=\frac{\beta_{3}^{*} \vartheta}{\rho l^{4}}
\end{aligned}
$$

The considered range for third-grade fluid parameter $\alpha_{3}$ and second-grade fluid parameters $\alpha_{1}, \alpha_{2}$ are taken from the inequality (Eq. (3)) and conditions of Eq. (9). Fosdick and Rajagopal [46] analyzed the thermodynamics and stability of third-grade fluid and concluded that boundedness and stability of third-grade fluids can be studied just as it was for second-grade fluid (examined by Dunn and Fosdick [47], for $\beta_{3}^{*}=0$ results in the constitutive relation for second-grade fluid). 
Fosdick and Straughan [48] showed that the condition $\alpha_{1}{ }^{*}<0$ gives non-physical results i.e., quite arbitrary flows with instability and unboundedness and asymptotic stability for $\alpha_{1}^{*} \geq 0$.

For higher $G r$ value, effect of the viscous force can be ignored in comparison to thermal buoyancy force (when $G r=1$ both viscous and thermal buoyancy contribute equally). Greater $G r$ influences the induced buoyancy force and mobilizes greater thermal convection effects Whereas at low values of Grashof number, the induced flow may be weak. Also, the range of Prandtl number is fixed as $\operatorname{Pr} \geq 1$, as it decides the thermal boundary layer thickness. $\operatorname{Pr}>1$ generally applies to denser liquids e.g. polymers for which thermal conductivity is significantly lower than air or water.

Following substitution in Eqns. (5a), (6) - (8), the non-dimensional governing equations and initial and boundary conditions emerge as:

$$
\begin{aligned}
& \frac{\partial U}{\partial X}+\frac{\partial V}{\partial Y}=0 \\
& \frac{\partial U}{\partial t}+U \frac{\partial U}{\partial X}+V \frac{\partial U}{\partial Y}=T+\frac{\partial^{2} U}{\partial Y^{2}}+\alpha_{1}\left[\frac{\partial^{3} U}{\partial t \partial Y^{2}}+\frac{\partial U}{\partial X} \frac{\partial^{2} U}{\partial Y^{2}}+U \frac{\partial^{3} U}{\partial X \partial Y^{2}}+V \frac{\partial^{3} U}{\partial Y^{3}}+3 \frac{\partial^{2} U}{\partial X \partial Y} \frac{\partial U}{\partial Y}\right] \\
& +\alpha_{2}\left[2 \frac{\partial U}{\partial Y} \frac{\partial^{2} U}{\partial X \partial Y}\right]+\alpha_{3}\left[6(G r)^{2}\left(\frac{\partial U}{\partial Y}\right)^{2} \frac{\partial^{2} U}{\partial Y^{2}}\right] \\
& \frac{\partial T}{\partial t}+U \frac{\partial T}{\partial X}+V \frac{\partial T}{\partial Y}=\frac{1}{P r}\left(\frac{\partial^{2} T}{\partial Y^{2}}\right) \\
& t=0: T=0, \quad V=0, \quad U=0 \quad \text { for all } X \text { and } Y \\
& t>0: T=1, \quad V=0, U=0 \quad \text { at } Y=0 \\
& T=0, \quad V=0, \quad U=0 \quad \text { at } X=0 \\
& T \rightarrow 0, \quad V \rightarrow 0, \quad U \rightarrow 0, \quad \frac{\partial U}{\partial Y} \rightarrow 0 \quad \text { as } Y \rightarrow \infty
\end{aligned}
$$


Employing an analytical method for the solution is a difficult task due to the strong non-linearity in the momentum and energy equations. Hence a computational method which is "unconditionally stable" known as the "Crank-Nicolson method" is deployed to solve Eqns. (10) - (12) using Eqn. (13). The "Crank-Nicolson" finite difference scheme is unconditionally stable and it does not require any stability condition for the numerical computations. Also, this method is compatible and stable. Hence, compatibility and stability ensure the convergence of results.

\subsection{Finite Difference Discretization}

The discretized finite difference equations corresponding to Eqns. (10), (11) and (12) are as follows:

$$
\begin{aligned}
& \frac{U_{f, g}^{h+1}-U_{f-1, g}^{h+1}+U_{f, g}^{h}-U_{f-1, g}^{h}}{2 \Delta X}+\frac{V_{f, g}^{h+1}-V_{f, g-1}^{h+1}+V_{f, g}^{h}-V_{f, g-1}^{h}}{2 \Delta Y}=0 \\
& \frac{U_{f, g}^{h+1}-U_{f, g}^{h}}{\Delta t}+\frac{U_{f, g}^{h}}{2 \Delta X}\left(U_{f, g}^{h+1}+U_{f, g}^{h}-U_{f-1, g}^{h+1}-U_{f-1, g}^{h}\right)+\frac{V_{f, g}^{h}}{4 \Delta Y}\left(U_{f, g+1}^{h+1}+U_{f, g+1}^{h}-U_{f, g-1}^{h+1}-U_{f, g-1}^{h}\right) \\
& =\frac{T_{f, g}^{h}+T_{f, g}^{h+1}}{2}++\frac{1}{2(\Delta Y)^{2}}\left(U_{f, g-1}^{h+1}+U_{f, g-1}^{h}-2 U_{f, g}^{h+1}-2 U_{f, g}^{h}+U_{f, g+1}^{h+1}+U_{f, g+1}^{h}\right)+ \\
& +\alpha_{1}\left[\frac{1}{4(\Delta Y)^{2}(\Delta t)}\left(U_{f, g-2}^{h+1}-U_{f, g-2}^{h}-2 U_{f, g}^{h+1}+2 U_{f, g}^{h}+U_{f, g+2}^{h+1}-U_{f, g+2}^{h}\right)\right] \\
& +\frac{\alpha_{1} U_{f, g}^{h}}{2(\Delta Y)^{2} \Delta X}\left[U_{f, g+1}^{h+1}+U_{f, g+1}^{h}-U_{f-1, g+1}^{h+1}-U_{f-1, g+1}^{h}-2 U_{f, g}^{h+1}-2 U_{f, g}^{h}+2 U_{f-1, g}^{h+1}+2 U_{f-1, g}^{h}+\right. \\
& \left.U_{f, g-1}^{h+1}+U_{f, g-1}^{h}-U_{f-1, g-1}^{h+1}-U_{f-1, g-1}^{h}\right] \\
& +\frac{\alpha_{1} V_{f, g}^{h}}{4(\Delta Y)^{3}}\left[U_{f, g+2}^{h+1}+U_{f, g+2}^{h}-2 U_{f, g+1}^{h+1}-2 U_{f, g+1}^{h}+2 U_{f, g-1}^{h+1}+2 U_{f, g-1}^{h}-U_{f, g-2}^{h+1}-U_{f, g-2}^{h}\right] \\
& +\frac{\alpha_{1}}{2(\Delta X)(\Delta Y)^{2}}\left(U_{f, g}^{h}-U_{f-1, g}^{h}\right)\left(U_{f, g-1}^{h+1}+U_{f, g-1}^{h}-2 U_{f, g}^{h+1}-2 U_{f, g}^{h}+U_{f, g+1}^{h+1}+U_{f, g+1}^{h}\right) \\
& +\frac{3 \alpha_{1}}{8(\Delta X)(\Delta Y)^{2}}\left(U_{f, g+1}^{h}-U_{f, g-1}^{h}\right)\left(U_{f, g+1}^{h+1}+U_{f, g+1}^{h}-U_{f-1, g+1}^{h+1}-U_{f-1, g+1}^{h}-U_{f, g-1}^{h+1}-\right. \\
& \left.U_{f, g-1}^{h}+U_{f-1, g-1}^{h+1}+U_{f-1, g-1}^{h}\right)
\end{aligned}
$$




$$
\begin{aligned}
& +\frac{\alpha_{2}}{4(\Delta Y)^{3}}\left(U_{f, g+1}^{h+1}+U_{f, g+1}^{h}-U_{f-1, g+1}^{h+1}-U_{f-1, g+1}^{h}-U_{f, g-1}^{h+1}-U_{f, g-1}^{h}+U_{f-1, g-1}^{h+1}+\right. \\
& \left.U_{f-1, g-1}^{h}\right)\left(U_{f, g+1}^{h}-U_{f, g-1}^{h}\right) \\
& +\frac{3 \alpha_{3}(G r)^{2}}{4(\Delta Y)^{4}}\left(U_{f, g+1}^{h}-U_{f, g-1}^{h}\right)^{2}\left(U_{f, g-1}^{h+1}+U_{f, g-1}^{h}-2 U_{f, g}^{h+1}-2 U_{f, g}^{h}+U_{f, g+1}^{h+1}+U_{f, g+1}^{h}\right) \\
& \frac{T_{f, g}^{h+1}-T_{f, g}^{h}}{\Delta t}+\frac{U_{f, g}^{h}}{2 \Delta X}\left(T_{f, g}^{h+1}-T_{f-1, g}^{h+1}+T_{f, g}^{h}-T_{f-1, g}^{h}\right)+\frac{V_{f, g}^{h}}{4 \Delta Y}\left(T_{f, g+1}^{h+1}-T_{f, g-1}^{h+1}+T_{f, g+1}^{h}-T_{f, g-1}^{h}\right) \\
& =\frac{1}{2 \operatorname{Pr}(\Delta Y)^{2}}\left(T_{f, g-1}^{h+1}-2 T_{f, g}^{h+1}+T_{f, g+1}^{h+1}+T_{f, g-1}^{h}-2 T_{f, g}^{h}+T_{f, g+1}^{h}\right)
\end{aligned}
$$

The selected flow domain is $X_{\min }=0, X_{\max }=1, Y_{\min }=0$ and $Y_{\max }=20$ (where $Y_{\max }$ implies to $Y=\infty)$.

Initially, the temperature field is computed using the finite difference thermal Eqn. (16). Then, the computational procedure continues to solve for the velocity field via the momentum (Eq. (15)) and mass conservation Eqn. (14). The tridiagonal and penta-diagonal forms of Eqns. (15) and (14) at $(h+1)^{\text {th }}$ stage are given as:

$$
\begin{aligned}
& A_{1} \delta_{f, g-1}^{h+1}+B_{1} \delta_{f, g}^{h+1}+C_{1} \delta_{f, g+1}^{h+1}=D_{1} \\
& A_{2} \gamma_{f, g-2}^{h+1}+B_{2} \gamma_{f, g-1}^{h+1}+C_{2} \gamma_{f, g}^{h+1}+D_{2} \gamma_{f, g+1}^{h+1}+E_{2} \gamma_{f, g+2}^{h+1}=F_{2}
\end{aligned}
$$

where $\delta$ and $\gamma$ specify $T$ and $U$, respectively. Hence, Eqns. (17) - (18) at each interior grid-point on a definite $f$-level comprise a system of equations in penta-diagonal and tridiagonal forms. A number of articles $[28,29]$ provide a more detailed explanation for this finite difference method.

\subsection{Validation of the numerical scheme:}

\subsubsection{Grid independency test:}

An economically stable consistent mesh is chosen by employing grid-independency tests for different mesh sizes. The Nusselt number $(\overline{N u})$ and average skin-friction coefficient $\left(\bar{C}_{f}\right)$ values 
for boundary condition $Y=0$ are given in Table 1. The grid-independency test implies that a mesh size of 100 X 500 yields a sufficient accuracy for the current problem. In the same way, a timeindependency test provides the best time-step size $\Delta t(t=h \Delta t, h=0,1,2, \ldots)$ for program execution and is documented in Table 2. For the present study, $\Delta t=0.01$ is therefore prescribed.

\subsubsection{Comparison studies:}

For the special case of Newtonian flow conditions $\left(\alpha_{1}=\alpha_{2}=\alpha_{3}=0\right)$ the present model reduces exactly to that investigated earlier by Takhar et al. [49] who employed power series expansions and Runge-Kutta-Merson numerical shooting methods. The comparison of the present finite difference solutions with Takhar et al. [49] is shown in Fig. 2(a). Also, the current numerical simulations are compared (in the absence of magnetic effects) with Abbas and Palani [50] (refer to Fig. 2(b)), who have considered a surface temperature gradient along with magnetic effect for a Newtonian fluid. Generally, a close correspondence is achieved confirming confidence in the present numerical code.

\section{RESULTS AND DISCUSSION}

The behaviour of the key flow-field variables (non-dimensional velocity $U$ and temperature $T)$ at different times are studied graphically. The flow $(U$ and $T)$ profiles are explained along with the direction of $Y$. The numerically simulated values give a detailed explanation for the variation of the non-dimensional quantities such as flow-variables, coefficients of heat transport and skinfriction along with Bejan number, entropy generation parameter for different controlling parameters. Extensive elaboration is provided in the following paragraphs.

\subsection{Velocity:}

The simulated transient non-dimensional velocity $(U)$ profiles at a specific location $(1,0.68)$ for various values of the viscoelastic fluid parameter $\left(\alpha_{1}\right)$, a second-grade fluid parameter $\left(\alpha_{2}\right)$, 
and third-grade fluid parameter $\left(\alpha_{3}\right)$ are depicted in Fig. 3. The $U$ profile, in the neighborhood of the heated vertical plate (Fig. 3) shows a particular trend, i.e., it initially grows with time to reach the peak value, thereafter exhibits a marginal fall and finally becomes independent of time tending to a constant value. Also, at the starting time $(t<<1)$, it is evident that the conductive heat transfer dominates over the convective heat transfer. Subsequently, after a particular time elapses, the heat transfer rate is influenced by natural convection alone and a significant acceleration of the thirdgrade fluid flow occurs with respect to time. It is also apparent in Fig. 3 that initially $(t<1)$ the unsteady $U$ profiles coincide with each other and only deviate at a later time $(t \geq 1)$. From Fig. 3 amplifying $\alpha_{1}$ or $\alpha_{3}$ both cause a decreasing trend in the velocity field $(U)$, whereas increasing $\alpha_{2}$ induces an increasing trend in velocity. Figure 3 also shows that the time needed to attain the maximum value is almost the same for variation of $\alpha_{2}$, whereas it increases when $\alpha_{1}$ or $\alpha_{3}$ rheological parameters are increased.

Figure 4 exhibits the evolution in steady-state velocity profile for the variations of $\alpha_{1}, \alpha_{2}$, and $\alpha_{3}$. Initially, the no-slip condition influences the $U$ curves to start with zero value, and then reach the maximum magnitude, after which velocity depletes to zero in the $Y$ direction in accordance with the edge (free stream) boundary conditions. Increasing $\alpha_{1}$ or $\alpha_{2}$, both elevate the time taken to attain the steady-state behaviour. However, the steady-state time is not tangibly modified with a variation of $\alpha_{3}$.

\subsection{Temperature}

The numerically generated non-dimensional temperature $(T)$ plots versus time are shown in Fig. 5 for different values of $\alpha_{1}, \alpha_{2}$, and $\alpha_{3}$ again at the location (1, 0.68). Initially, there is observed to be a drastic increase in the $T$ curves which all ascend to their peaks, then decreases slightly, and finally attain the time-independent state. As $\alpha_{1}, \alpha_{2}$ and $\alpha_{3}$ increases, time for attaining peak value increases. Increasing third-grade parameter values, therefore energize the regime and give higher temperature values. It is evident that the viscoelastic effect of third-grade parameter encourages 
collision of the fluid particles and increases the fluid temperature in the vicinity of the hot plate and simultaneously (owing to higher viscosity) decelerates the boundary layer flow.

The impact of rheological parameters $\alpha_{1}, \alpha_{2}$ and $\alpha_{3}$ on the time-independent $T$ plots against $Y$ are depicted in Fig. 6. All $T$ curves start with $T=1$, i.e., boundary condition maintained at the hot plate then follow a monotonic decrease eventually decaying to zero in the free stream. As the effect of viscoelastic property of third-grade parameter increases, this enhances the thermal boundary layer thickness due to amplified temperature values. It also modifies the time taken to attain the steady-state.

\subsection{Friction and Heat Transport Coefficients}

For the numerically generated known third-grade flow-field variables associated with both unsteady and steady-state conditions, the coefficients of heat transport rate $(\overline{N u})$ and friction $\left(\overline{C_{f}}\right)$ are critical parameters. They provide important wall characteristics in convective heat transfer problems. Here, their non-dimensional forms are given by

$\overline{C_{f}}=\int_{0}^{1}\left(\frac{\partial U}{\partial Y}\right)_{Y=0} d X$ and $\overline{N u}=-\int_{0}^{1}\left(\frac{\partial T}{\partial Y}\right)_{Y=0} d X$

The calculation procedure for these coefficients involves the 5-point approximation formulae and Newton-Cotes quadrature.

For the variations of $\alpha_{1}, \alpha_{2}$ and $\alpha_{3}$, Fig. 7 visualizes the distributions for the coefficient of friction $\left(\overline{C_{f}}\right)$ and Fig.8 illustrates the variation in the heat transfer coefficient. Inspection of Fig. 7 shows that at the initial stage, the $\overline{C_{f}}$ curves ascend with time to a maximum value then decrease to lower values. Following this, all curves reach the asymptotic time-independent state. Skinfriction is an increasing function of $\alpha_{2}$ whereas it is a decreasing function of $\alpha_{1}$ or $\alpha_{3}$. As we increase the third-grade parameter, $\alpha_{3}$, the viscoelastic property of the fluid increases and hence hinders the flow past a wall, as a result, shearing is reduced, and low-velocity values are observed at the hot wall. 
Figure 8 shows the results for heat transfer rate $(\overline{N u})$ at the hot surface for the third-grade free convection flow (i.e. at $Y=0$ ). At the starting time, the $\overline{N u}$ curves fall to significantly smaller values, increase and then finally attain the steady-state with further time elapse. The $\overline{N u}$ values increase for decreasing third-grade parameter $\left(\alpha_{3}\right)$. For increased $\alpha_{3}$, particle collision increases and this manifests in an increased flow temperature and hence negatively increased $\overline{N u}$ values are observed in Fig. 8. Higher $\overline{N u}$ values imply greater transfer of heat to the wall from the boundary layer.

\subsection{Entropy generation analysis}

The exchange of thermal energy and the momentum due to viscous dissipation and heat transfer result in an imbalanced condition in the fluid medium, and at the boundary. Hence there will be a continuous generation of entropy. For incompressible non-Newtonian third-grade fluid, this entropy generation per unit volume is expressed as [51-56]:

$$
\begin{aligned}
& E_{\text {gen }}=\frac{k}{{T_{\infty}^{\prime}}^{2}}\left(\frac{\partial T^{\prime}}{\partial \bar{y}}\right)^{2}+\left\{\frac{\mu}{T_{\infty}^{\prime}}\left(\frac{\partial \bar{u}}{\partial \bar{y}}\right)^{2}+\frac{\alpha_{1}^{*}}{T_{\infty}^{\prime}}\left[\frac{\partial^{2} \bar{u}}{\partial t^{\prime} \partial \bar{y}} \frac{\partial \bar{u}}{\partial \bar{y}}+\bar{u} \frac{\partial^{2} \bar{u}}{\partial \bar{x} \partial \bar{y}} \frac{\partial \bar{u}}{\partial \bar{y}}+\bar{v} \frac{\partial^{2} \bar{u}}{\partial \bar{y}^{2}} \frac{\partial \bar{u}}{\partial \bar{y}}\right]+\frac{\beta_{3}^{*}}{T_{\infty}^{\prime}}\left[2\left(\frac{\partial \bar{u}}{\partial \bar{y}}\right)^{4}\right]\right\} \\
& E_{\text {gen }}=E_{1}+E_{2}
\end{aligned}
$$

where

$$
\begin{gathered}
E_{1}=\frac{k}{{T_{\infty}^{\prime}}^{2}}\left(\frac{\partial T^{\prime}}{\partial \bar{y}}\right)^{2} \text { (Due to heat transfer) } \\
E_{2}=\left\{\frac{\mu}{T_{\infty}^{\prime}}\left(\frac{\partial \bar{u}}{\partial \bar{y}}\right)^{2}+\frac{\alpha_{1}^{*}}{T_{\infty}^{\prime}}\left[\frac{\partial^{2} \bar{u}}{\partial t^{\prime} \partial \bar{y}} \frac{\partial \bar{u}}{\partial \bar{y}}+\bar{u} \frac{\partial^{2} \bar{u}}{\partial \bar{x} \partial \bar{y}} \frac{\partial \bar{u}}{\partial \bar{y}}+\bar{v} \frac{\partial^{2} \bar{u}}{\partial \bar{y}^{2}} \frac{\partial \bar{u}}{\partial \bar{y}}\right]+\frac{\beta_{3}^{*}}{T_{\infty}^{\prime}}\left[2\left(\frac{\partial \bar{u}}{\partial \bar{y}}\right)^{4}\right]\right\}
\end{gathered}
$$

(Due to viscous effect of incompressible third-grade fluid)

The dimensionless parameter for entropy generation $N s$ is given as,

$$
N s=\frac{E_{\text {gen }}}{E_{\text {char }}}
$$


By defining $E_{\text {char }}=\frac{k\left(T_{w}^{\prime}-T_{\infty}^{\prime}\right)}{T_{\infty}^{\prime 2} l^{2}}($ characteristic entropy generation $)$ and $\Omega=\frac{\left(T_{w}^{\prime}-T_{\infty}^{\prime}\right)}{T_{\infty}^{\prime}}($ dimensionless temperature difference) we obtain:

$$
\begin{aligned}
& \qquad N s=\left(\frac{\partial T}{\partial Y}\right)^{2}+\frac{\operatorname{Br}(\mathrm{Gr})^{2}}{\Omega}\left\{\left(\frac{\partial U}{\partial Y}\right)^{2}+\alpha_{1}\left[\frac{\partial^{2} U}{\partial t \partial Y} \frac{\partial U}{\partial Y}+U \frac{\partial^{2} U}{\partial X \partial Y} \frac{\partial U}{\partial Y}+V \frac{\partial^{2} U}{\partial Y^{2}} \frac{\partial U}{\partial Y}\right]+\beta\left[2(\mathrm{Gr})^{2}\left(\frac{\partial U}{\partial Y}\right)^{4}\right]\right\} \\
& N s=N_{1}+N_{2}=\text { Due to heat transfer }+ \text { viscous effect } \\
& \text { i.e., } \\
& \qquad N_{1}=\left(\frac{\partial T}{\partial Y}\right)^{2} \\
& \qquad N_{2}=\frac{\operatorname{Br}(\mathrm{Gr})^{2}}{\Omega}\left\{\left(\frac{\partial U}{\partial Y}\right)^{2}+\alpha_{1}\left[\frac{\partial^{2} U}{\partial t \partial Y} \frac{\partial U}{\partial Y}+U \frac{\partial^{2} U}{\partial X \partial Y} \frac{\partial U}{\partial Y}+V \frac{\partial^{2} U}{\partial Y^{2}} \frac{\partial U}{\partial Y}\right]+\beta\left[2(\mathrm{Gr})^{2}\left(\frac{\partial U}{\partial Y}\right)^{4}\right]\right\}
\end{aligned}
$$

Here Brinkman number $(\mathrm{Br})$ accounts for viscosity dissipation due to the friction of fluid particles corresponding to conductive heat flow which is the result of temperature difference. Also, $B r \Omega^{-1}$ is one more significant non-dimensional number which is referred to as the group parameter in the analysis of entropy generation. Here, $B r \Omega^{-1}>0$ signifies the effect of viscosity mainly due to the fluid. While studying the effect of entropy generation it is important to consider viscous effects.

The parameter $B e$ (Bejan number) is introduced for the calculation of irreversibility, and may be defined as the quantitative relationship between the generation of entropy due to heat flow to the total entropy production i.e.,

$B e=\frac{N_{1}}{N_{1}+N_{2}}$

The parameter $B e$ (Bejan number) belongs to a closed interval of $0 \& 1$, i.e., $0 \leq B e \leq 1$. Any particular $B e$ number within the range indicates the dominating effect of different factors causing entropy heat generation. Accordingly, different cases can be studied, as listed below:

Case 1. $B e=0$; The parameter $N_{2}$ dominates the parameter $N_{1}$

Case 2. $B e=1$; The parameter $N_{1}$ dominates the parameter $N_{2}$. 
Case 3. $B e=0.5$; Both irreversibility (heat transfer \& fluid friction) have the same impact on entropy generation production (i.e., $N_{2}=N_{1}$ ).

\subsection{Results of entropy generation analysis}

It is essential to discuss the effect of various non-dimensional numbers for calculation of $N s$ and $\mathrm{Be}$ at both transient and steady conditions for free-convective flow. In the present paper, the influence of Gr (Grashof number), $\operatorname{Pr}$ (Prandtl number), Br $\Omega^{-1}$ (group parameter) and effect of a third-grade parameter $\left(\alpha_{3}\right)$ are shown graphically.

\section{5-1 Effect of $\alpha_{1}, \alpha_{2}$ and $\alpha_{3}$ (third-grade fluid parameters)}

The influence of $\alpha_{1}, \alpha_{2}$, and $\alpha_{3}$ i.e. the viscoelastic third-grade fluid parameters on the calculation of entropy heat generation parameter and Bejan number under steady and unsteady conditions are deliberated through graphs and described below.

\section{Behaviour of entropy generation number $(\mathrm{Ns})$}

From transient graph (Fig. 9(a)), for $t<1.5$ heat conduction is dominated. In Fig. 9(a), as $\alpha_{1}$ or $\alpha_{2}$ increases, the $N s$ parameter increases whereas when $\alpha_{3}$ increases, the $N s$ parameter decreases. It is witnessed that intensified $\alpha_{3}$ implies higher viscoelastic properties of the third-grade fluid which results in the stronger bonding forces between the fluid particles in the free-convective laminar flow. Therefore, the amount of heat released is absorbed to excite the fluid particles. This results in a decrease in entropy generation which is presented through Fig. 9(a). Again, the steadystate profile for $N s$ (refer to Fig. 9(b)) shows that $N s$ is decreased by increasing $\alpha_{3}$ and here also a similar explanation can support the result. Also, the steady-state time increases with increasing $\alpha_{1}$ or $\alpha_{2}$ or $\alpha_{3}$.

\section{Behaviour of Bejan number $(B e)$}


Figures 10(a), (b) illustrate the impact on $B e$ of the rheological parameters, $\alpha_{1}, \alpha_{2}$ and $\alpha_{3}$. Figure 10(a) gives transient numerical $B e$ values for different $\alpha_{1}, \alpha_{2}$, and $\alpha_{3}$. Bejan number is enhanced with an elevation in $\alpha_{2}$ or $\alpha_{3}$ whereas it is reduced with greater $\alpha_{1}$. As $\alpha_{3}$ intensifies, viscoelastic behavior of third-grade fluid increases and this modification in viscous and elastic properties results in an increase in Bejan number $(B e)$ values. $B e$ lines almost coincide for variations of $\alpha_{1}$, $\alpha_{2}$, and $\alpha_{3}$ in the steady-state plots (Fig. 10(b)) and Be number is decreasing for increasing $\alpha_{3}$. Former observation is noticed since fluid flow is influenced by heat transfer as it is remarked that Bejan number value is greater than 0.5 for variation of $\alpha_{3}$ immediately adjacent to the hot plate. After reaching the steady-state condition, it is apparent that Bejan number decreases as $\alpha_{1}$ or $\alpha_{2}$ or $\alpha_{3}$ decrease. The steady-state time is also observed to be accentuated with escalating values of $\alpha_{1}$ or $\alpha_{2}$ or $\alpha_{3}$.

\section{5-2 Effect of $\mathrm{Br} \Omega^{-1}, \mathrm{Gr}$ and $\mathrm{Pr}$}

\section{Behaviour of entropy generation number (Ns)}

The variation of $B r \Omega^{-1}, G r$ and $\operatorname{Pr}$ all show a significant effect on entropy heat generation which is evaluated numerically by the non-dimensional parameter $N S$ for the unsteady state and these results are depicted in Figs. 11(a) and 11(b) respectively. The non-dimensional Grashof number, $G r$ signifies the relative influence of thermal buoyancy force to viscous hydrodynamic force in buoyancy-driven (natural convective) fluid-flow. Figure 11(a) presents the effect of $\mathrm{Br} \Omega^{-1}$ and $\mathrm{Gr}$ on non-dimensional $N s$ under an unsteady condition at constant $\mathrm{Pr}$. From Fig. 11(a), it is evident that $N s$ is increasing for increasing values of $B r \Omega^{-1}$ or $G r$. The induced buoyancy forces are more for increased $G r$ which contributes to higher temperature differences and hence an enhanced rate of heat transfer. This may be the reason for increased entropy generation. $\mathrm{Br} \Omega^{-1}$ signifies the effect of viscosity mainly due to the fluid. This parameter is related to fluid viscosity term and appears with velocity components (referring Eq. 26). Hence an increase in group parameter increases the velocity of the fluid which in turn results in higher entropy generation. More fluctuations can be observed near the heated plate, which may be 
attributable to the frictional effect of fluid particles at the boundary. The time taken to reach maximum $N s$ is almost same for several values of each physical parameter. Figure 11(b) denotes influence of $\mathrm{Gr}$ and $\mathrm{Pr}$ on unsteady $\mathrm{Ns}$ profiles for constant $\mathrm{Br} \Omega^{-1}$. A significant observation can be made from Fig. 11(b) that the entropy curves are overlapped with each other for increasing $G r$ values. However, in particular, it is seen that for larger values of $G r$, the $N s$ profiles show an increasing trend i.e. thermal buoyancy encourages entropy generation. Also, a variation of $P r$ substantially alters the free-convective flow of the third-grade fluid. Here, Ns curves increase monotonically, then show some oscillations and finally reach the steady-state. Moreover, for increased $\operatorname{Pr}$, the peak value of the dimensionless entropy heat generation parameter of the thirdgrade fluid is almost the same. The $N s$ profile has an increasing trend with increasing $\operatorname{Pr}$ number i.e. with decreasing thermal conductivity. This shows that with decreasing $\operatorname{Pr}$ (increasing thermal conductivity) the entropy heat generation can be minimized for third-grade fluids.

Figures 12(a)-12(b) show the steady-state effects of $G r, B r \Omega^{-1}$ and $\operatorname{Pr}$ on $N s$. Initially, $N s$ shows an increasing trend and then sharply decreases for variation of $\mathrm{Gr}$ and $\mathrm{Br} \Omega^{-1}$ due to dominant effects of heat transfer (near the plate) and fluid friction (away from the plate) respectively. For almost at $Y>5.63$, the steady-state condition is reached. The maximum Ns value is the increasing function of both $\mathrm{Gr}$ and $\mathrm{Br} \Omega^{-1}$. The effect of $\mathrm{Br} \Omega^{-1}$ on entropy generation is more in comparison with $\mathrm{Gr}$. This is a significant result in the minimization of entropy heat generation. Also, amplifying $G r$ and $B r \Omega^{-1}$, the steady-state time is almost the same. The steadystate plots from Fig. 12(b) give numerically generated $N s$ values for different $P r$ and $G r$. It is seen that for different values of $P r$ and $G r, N s$ curves increase from zero value to a maximum then abruptly fall to zero. Here, the curves are almost overlapped for the variation of parameter values. Initially in the third-grade fluid region (i.e., $0<Y<1.63$ ), $N s$ profiles show an increasing trend, and for $Y>1.63$ this trend is reversed exactly with greater $\operatorname{Pr}$ values. It is evident that for 
increasing $\mathrm{Gr}$ the curves show an increasing trend. Also, the steady-state time is largely invariant to a change in $G r$ as compared to $P r$.

\section{Behaviour of Bejan number $(B e)$}

Figures 13(a) and 13(b) show results for the impact of different physical parameters on Bejan number at the transient condition. From Fig. 13(a) for different $\mathrm{Gr}$ or $\mathrm{Br} \Omega^{-1}$, at the time-dependent condition the $B e$ lines begin with small fluctuations, increase monotonically, an again exhibit some oscillations before reaching the steady-state. The $B e$ show the increasing trend for varying values of $\mathrm{Gr}$ or $\mathrm{Br} \Omega^{-1}$. Furthermore, the time needed to attain the maximum Bejan number value is almost the same for the variation of the different parameters. The $B e$ profile for variation of $G r$ is higher than the variation of $\mathrm{Br} \Omega^{-1}$. Figure 13(b) reveals interesting trends regarding the effects of $G r$ or $\operatorname{Pr}$ on Bejan number under the unsteady condition. For the chosen values of $\operatorname{Pr}$, the maximum $B e$ values are small, but the effect of $\operatorname{Pr}$ on $B e$ is considerable. As $\operatorname{Pr}$ increases, $B e$ decreases. Also, the time required for reaching the maximum value amplifies with increased $\operatorname{Pr}$. However at a constant value of $\operatorname{Pr}$ and for variation of $G r$, this is almost the same. In both transient graphs for $B e$ in the vicinity of the heated plate, the entropy generation due fluid friction dominates over heat transfer since $0<B e<0.5$.

The steady-state results of $B e$ for several parameter values are reflected in Figs. 14(a) -14(b). All $B e$ curves for different values of $\mathrm{Gr}$ or $\mathrm{Br} \Omega^{-1}$ show a similar trend. Initially, these curves increase from $B e=0\left(N_{2}>N_{1}\right)$ to reach maximum value along $Y$ direction then suddenly decrease to zero almost at $Y=1.42$. Again for $Y>1.99 \mathrm{Be}$ values start to increase slightly to maintain the same trend thereafter (at $Y>6.11$ ) reaching zero values (refer to Fig. 14(a)). For increased $G r$ or $B r \Omega^{-1}, B e>0.5$ i.e., heat transfer influences the entropy generation near the hot plate which is depicted in Fig. 14(a). For smaller $B r \Omega^{-1}$, irreversibility due to the viscous effect exceeds that due to heat transfer. Also, in the flow region far away from the plate irreversibility due to viscous effect dominates over the heat transfer. Here, it is noted that steady-state time is not varying much with 
group parameter or Grashof number. Figure 14(b) depicts the steady-state plot for $B e$ for different values of $\operatorname{Pr}$ or $G r$. The $B e$ curves for increased $\operatorname{Pr}$ start from zero, reach peak value and then sharply fall to zero (almost at $Y=1.41$ ). They exhibit small oscillation and then attain zero value for $Y>$ 5.78. As $\operatorname{Pr}$ increases (lower thermal conductivity) the Bejan lines show a decreasing trend and the maximum attained $B e$ is less than 0.2 near the plate i.e., irreversibility caused by frictional effects due to the fluid is elevated. Here also (at constant $\operatorname{Pr}$ condition), $B e$ profile shows an increasing trend with greater values of $G r$.

\section{5-3 Entropy and Bejan contours:}

Figures 15 (a)-(d) present the entropy lines in a two-dimensional plane $(X, Y)$ for different values of $\mathrm{Gr}$ (Grashof number), $\operatorname{Pr}$ (Prandtl number), Br $\Omega^{-1}$ (group parameter) and third-grade parameter $\left(\alpha_{3}\right)$. It is observed that the entropy lines are typically concentrated near the heated plate. From Figs. 15 (a), (b) \& (c), as $\mathrm{Gr}, \operatorname{Pr}$ and $B r \Omega^{-1}$ increase, the entropy lines are moving away from the hot plate, respectively. In the same way, from Fig. 15 (d), as $\alpha_{3}$ increases, the entropy lines move a little distance from the plate. These observations are consistently witnessed at any point of the two-dimensional third-grade fluid free convection flow-domain.

Likewise, Figs. 16 (a) \& (d) show the results for Bejan lines in the whole flow-domain for the third-grade fluid. For increased $G r, B r \Omega^{-1}$ and $\alpha_{3}$, the Bejan lines are moving away from the plate, as is evident from Figs. 16 (a), (c) \& (d), respectively. Also, these lines move nearer to the hot plate i.e. they become constricted, as $\operatorname{Pr}$ increases (refer to Fig. 16 (b). Immediately adjacent to the heated plate $B e$ contour lines show that $B e$ number is increasing for increased $\mathrm{Gr}, \mathrm{Br} \Omega^{-1}$ and is greater than 0.5 i.e., heat transfer influences the entropy generation. However, for amplifying $\operatorname{Pr}$ or $\alpha_{3}$, exactly the reverse observation is noticed.

\section{5-4 Comparison contour plot}


The contours for flow-variables $(U \& T)$ of the third-grade fluid (Fig. 17 (a)) and Newtonian fluid (Fig. 17 (b)) are visualized in the two-dimensional rectangular plane $(0<X<1,0<Y<$ 2). The contour lines for the third-grade fluid tend to deviate more as compared to Newtonian fluid from the heated vertical plate. Also, the shapes of contours vary slightly. These patterns demonstrate clearly that computed results for third-grade fluid vary significantly with respect to the Newtonian fluid.

\section{Concluding Remarks}

Thermodynamic entropy generation minimization analysis has been conducted for transient third-grade fluid free convection flow from the semi-infinite heated vertical plate. The normalized unsteady boundary layer equations for mass, momentum, and energy (heat) conservation have been solved by a numerical Crank-Nicolson finite difference scheme. With known flow-variables (temperature and velocity), the entropy heat generation parameter and Bejan number are calculated. The effect of different control parameters i.e. Grashof number (Gr), Prandtl number $(\operatorname{Pr})$, group parameter $\left(B r \Omega^{-1}\right)$ and rheological third-grade parameter $\left(\alpha_{3}\right)$ on entropy analysis are discussed. Validation of the finite difference numerical code is conducted with gridindependence and time-independence tests and also with earlier Newtonian results from the literature. The major findings of the current study can be listed as:

- As third-grade parameter intensifies temperature rises and velocity decreases.

- As third-grade parameter increases both skin-friction and heat transfer coefficient decrease.

- Entropy heat generation increases for increasing values of Grashof number or Prandtl number or group parameter (Brinkman number divided by temperature difference) whereas it decreases with increasing third-grade rheological parameter.

- Bejan number is enhanced with increasing Grashof number or group parameter or thirdgrade rheological parameter whereas it is depressed with increasing Prandtl number. 
- For the first time, entropy generation contours and the Bejan number contours are displayed in the flow-domain for the third-grade fluid flow.

The present work has considered the Reiner-Rivlin third-grade non-Newtonian differential model. Future studies will consider alternative rheological models including the Oldroyd-B model [57], single-phase and two-phase Jeffery's viscoelastic models $[58,59]$ and may also consider nonFourier heat flux models in rheological coating heat transfer [60].

\section{REFERENCES:}

1. L. Prandtl 'Uber Flussigkeitsbewegung bei sehr kleiner Reibung', Verh, III. Int Math Kongr Heidelberg. Leipzig: Teubner; (1904) 484-91.

2. H. Blasius 'Grenzschiechten in Flussigkeiten mit kleiner Reibung', Z Math U Phys [(English translation) NACATM 1256] 56 (1908) 1-37.

3. J.H. Merkin 'A note on the similarity solutions for free convection on a vertical plate', $J$. Eng. Math, 19, 189-201 (1985).

4. M. A. Mansour 'Radiative and free-convection effects on the oscillatory flow past a vertical plate', Astrophysics and Space Science 166 (1990) 269-275.

5. H. S. Takhar, Rama Subba Reddy Gorla, V.M. Soundalgekar 'Radiation effects on MHD free convection flow of a gas past a semi-infinite vertical plate', International Journal of Numerical Methods for Heat \& Fluid Flow 6(2) (1996) 77 - 83.

6. A.V. Kuznetsov, D.A. Nield 'Natural convective boundary-layer flow of a nanofluid past a vertical plate', International Journal of Thermal Sciences 49 (2010) 243-247.

7. A.V. Kuznetsov, D.A. Nield 'Natural convective boundary-layer flow of a nanofluid past a vertical plate: A revised model', International Journal of Thermal Sciences 77 (2014) 126-129. 
8. R. Biswas, M. Afikuzzaman M. Mondal, S.F. Ahmmed, 'MHD free convection and heat transfer flow through a vertical porous plate in the presence of chemical reaction', Frontiers in Heat and Mass Transfer, Vol. 11(1), 2018.

9. Md. Afikuzzaman, M. Ferdows, Md. Mahmud Alam,'Unsteady MHD Casson fluid flow through a parallel plate with hall current', Procedia Engineering, Volume 105, pp. 287293, 2015.

10. G. Janardhana Reddy, Bhasker reddy, J. C. Umavathi, Mikhail A. Sheremet,'Heat flow visualization for unsteady Casson fluid past a vertical slender hollow cylinder,, Thermal Science and Engineering Progress 5 (2018) 172-181.

11. G. Janardhana Reddy, M. Kumar, B. Kethireddy, A. J. Chamkha 'Colloidal study of unsteady magnetohydrodynamic couple stress fluid flow over an isothermal vertical flat plate with entropy heat generation', Journal of Molecular Liquids 252 (2018) 169-179.

12. M. Ayub, A. Rasheed, T. Hayat 'Exact flow of a third-grade fluid past a porous plate using homotopy analysis method', International Journal of Engineering Science 41 (2003) 2091-2103.

13. Valentina Busuioca, Dragos Iftimie 'Global existence and uniqueness of solutions for the equations of third grade fluids', International Journal of Non-Linear Mechanics 39 (2004) $1-12$.

14. F. Talay Akyildiz, Hamid Bellout and K. Vajravelu 'Exact solutions of nonlinear differential equations arising in third grade fluid flows', International Journal of NonLinear Mechanics 39 (2004) 1571-1578.

15. M. Sajid, T. Hayat and S. Asghar 'Non-similar analytic solution for MHD flow and heat transfer in a third-order fluid over a stretching sheet', International Journal of Heat and Mass Transfer 50 (2007) 1723-1736. 
16. O Anwar Bég, H S Takhar, R Bhargava, S Rawat and V R Prasad 'Numerical study of heat transfer of a third-grade viscoelastic fluid in a non-Darcy porous media with thermophysical effects', Physica Scripta 77 (2008) 065402-13.

17. B. Sahoo 'Hiemenz flow and heat transfer of a third grade fluid', Communications in Nonlinear Science and Numerical Simulation 14(3) (2009) 811-826.

18. B. Sahoo and Y. Do 'Effect of slip on sheet-driven flow and heat transfer of a third-grade fluid past a stretching sheet', International Communications in Heat and Mass Transfer 37 (2010) 1064-1071.

19. B. Sahoo and S. Poncet 'Flow and Heat Transfer of a third-grade fluid past an exponentially stretching sheet with partial slip boundary condition', International Journal of Heat and Mass Transfer 54(23-24) (2011) 5010-5019.

20. M. Keimanesh, M.M. Rashidi, Ali J. Chamkha, R. Jafari 'Study of a third-grade nonNewtonian fluid flow between two parallel plates using the multi-step differential transform method', Computers \& Mathematics with Applications 62(8) (2011) 2871-2891.

21. I.G. Baoku, B.I. Olajuwon, A.O. Mustapha 'Heat and mass transfer on a MHD third grade fluid with partial slip flow past an infinite vertical insulated porous plate in a porous medium', International Journal of Heat and Fluid Flow 40 (2013) 81-88.

22. T. Hayat, S. A. Shehzad, M. Qasim, S. Asghar and A. Alsaedi 'Thermally stratified radiative flow of third grade fluid over a stretching surface', AIAA Journal of Thermophysics and Heat Transfer 28(1) (2014) 155-161.

23. S. Nadeem and S Saleem 'Analytical study of third grade fluid over a rotating vertical cone in the presence of nanoparticles', International Journal of Heat and Mass Transfer 85 (2015) 1041-1048.

24. S. O. Adesanya and O. D Makinde 'Thermodynamic analysis for a third-grade fluid through a vertical channel with internal heat generation', Journal of Hydrodynamics 27(2) (2015) 264-272. 
25. T. Latif, N. Alvi, Q. Hussain and S. Asghar 'Variable properties of MHD third order fluid with peristalsis', Results in Physics 6 (2016) 963-972.

26. Z. Abbas, T. Javed, N. Ali and M. Sajid 'Diffusion of chemically reactive species in stagnation-point flow of a third-grade fluid: a hybrid numerical method', Journal of Applied Fluid Mechanics 9 (2016) 195-203.

27. T. Hayat, I. Ullah, T. Muhammad, A. Alsaedi 'A revised model for stretched flow of third grade fluid subject to magneto nanoparticles and convective condition', Journal of Molecular Liquids 230 (2017) 608-615.

28. G.J. Reddy, A. Hiremath, and M. Kumar 'Computational modeling of unsteady third-grade fluid flow over a vertical cylinder: a study of heat transfer visualization', Results in Physics 8 (2018) 671-682.

29. G.J. Reddy, A. Hiremath, Hussain Basha and N.S. Venkata Narayanan 'Transient flow and heat transfer characteristics of non-Newtonian supercritical third-grade fluid $\left(\mathrm{CO}_{2}\right)$ past a vertical cylinder', International Journal of Chemical Reactor Engineering (2018) 20170232.

30. S. Carnot 'Réflexions sur la puissance motrice du feu, et sur les machines propres à dèvelopper cette puissance', Paris, France (1824).

31. A. Bejan 'A Study of Entropy generation in fundamental convective heat transfer', ASME J. Heat Transfer 101 (4) (1979) 718.

32. A. Bejan 'Second law analysis in heat transfer', Energy 5 (8-9) (1980) 720-732.

33. A. Bejan 'Entropy generation through heat and fluid flow', Wiley, New York, USA (1982).

34. A. Bejan 'Entropy generation minimization: the new thermodynamics of finite-size devices and finite-time processes', Journal of Applied Physics 79 (1996) 1191-1218.

35. A. Bejan 'Fundamentals of exergy analysis, entropy generation minimization, and the generation of flow architecture', International Journal of Energy Research 26 (2002) 545565. 
36. O. D. Makinde and O. Anwar Bég 'On inherent irreversibility in a reactive hydromagnetic channel flow', Journal of Thermal Science 19(1) (2010) 72-79.

37. M. Magherbi, H. Abbassi and A. Ben Brahim 'Entropy generation at the onset of natural convection', International Journal of Heat and Mass Transfer 46 (2003) 3441-3450.

38. S. Mahmud and R. A. Fraser 'The second law analysis in fundamental convective heat transfer problems', International Journal of Thermal Sciences 42 (2003) 177-186.

39. Mikhail A. Sheremet, Hakan F. Öztop, loan Pop, Nidal Abu Hamdeh, ‘Analysis of entropy generation in natural convection of nanofluid inside a square cavity having hot solid block: Tiwari and Das' model, Entropy 18 (9) (2016) doi:10.3390/e18010009.

40. Nadezhda S. Bondareva, Mikhail A. Sheremet, Hakan F. Oztop, Nidal Abu-Hamdeh, 'Entropy generation due to natural convection of a nanofluid in a partially open triangular cavity, Advanced Powder Technology 28 (2017) 244-255.

41. G. Janardhana Reddy, Mahesh Kumar, J.C. Umavathi, Mikhail A. Sheremet,'Transient entropy analysis for the flow of a second grade fluid over a vertical cylinder, Canadian Journal of Physics 96 (2018) 978-991.

42. Giorgio Giangaspero, Enrico Sciubba, 'Application of the entropy generation minimization method to a solar heat exchanger: A pseudo-optimization design process based on the analysis of the local entropy generation maps', Volume 58, 1 September 2013, Pages 5265.

43. Toni Eger, Thomas Bol, Ayothi Ramanathan Thanu, László Darócz, Gábor Janiga 2, Rüdiger Schroth and Dominique Thévenin, 'Application of entropy generation to improve heat transfer of heat sinks in electric machines', Entropy 2017, 19, 255.

44. Xue-Tao Cheng and Xin-Gang Liang, 'Role of entropy generation minimization in thermal optimization', Chin. Phys. B Vol. 26, No. 12 (2017) 120505. 
45. Elisa Guelpa and Vittorio Verda, 'Entropy Generation Analysis of Wildfire Propagation', Entropy 2017, 19, 433.

46. R. L. Fosdick, and K. R. Rajagopal 'Thermodynamics and stability of fluids of third grade', Proceedings of the Royal Society of London. Series A, 369 (1980) $351-377$.

47. Dunn, J. E. and Rajagopal, K. R. 'Fluids of differential type: critical review and thermodynamic analysis', International Journal of Engineering Science, 33, pp. 689 - 729 (1995).

48. R. L. Fosdick and B. Straughan, 'Catastrophic instabilities and related results in a fluid of third grade', Int. J. Non-Linear Mech. 16, 191(1981).

49. H.S. Takhar, P. Ganesan, K. Ekambavanan and V.M. Soundalgekar 'Transient free convection pasta semi-infinite vertical plate with variable surface temperature', International Journal for Numerical Methods for Heat \& Fluid Flow 7(4) (1997) 280-296.

50. Ibrahim A. Abbas and G. Palani 'Effects of magnetohydrodynamic flow past a vertical plate with variable surface temperature', Applied Mathematics and Mechanics -Engl. Ed. 31(3) (2010) 329-338.

51. S. O. Adesanya and J.A. Falad 'Thermodynamics analysis of hydromagnetic third grade fluid flow through a channel filled with porous medium', Alexandria Engineering Journal $54(2015) 615-622$.

52. B. S. Yilbas and M. Pakdemirli 'Entropy generation due to the flow of a non-Newtonian fluid with variable viscosity in a circular pipe', Heat Transfer Engineering, 26(10) (2005) $80-86$.

53. M. Pakdemirli and B. S. Yilbas 'Entropy generation in a pipe due to non-Newtonian fluid flow: Constant viscosity case', Sadhana 31 (2006) 21-29. 
54. M. Pakdemirli and B.S. Yilbas 'Entropy generation for pipe flow of a third grade fluid with Vogel model viscosity', International Journal of Non-Linear Mechanics 41 (2006) 432437.

55. D. S. Chauhan and V. Kumar 'Entropy analysis for third-grade fluid flow with temperaturedependent viscosity in annulus partially filled with porous medium', Theor. Appl. Mech. 40 (2013) 441.

56. S. O. Adesanya, J.A. Falade, Srinivas Jangili and O. Anwar Bég 'Irreversibility analysis for reactive third-grade fluid flow and heat transfer with convective wall cooling', Alexandria Engineering Journal 56 (2017) 153-160.

57. M. Norouzi, M. Davoodi and O. Anwar Bég, MD. Shamshuddin, 'Theoretical study of Oldroyd-B visco-elastic fluid flow through curved pipes with slip effects in polymer flow processing', Int. J. Applied Computational Mathematics (2018). doi.org/10.1007/s40819018-0541-7 (22 pages).

58. V K Narla, Dharmendra Tripathi, O. Anwar Bég and A Kadir, 'Modelling transient magnetohydrodynamic peristaltic pumping of electroconductive viscoelastic fluids through a deformable curved channel', Journal of Engineering Mathematics (2018). https://doi.org/10.1007/s10665-018-9958-6 (17 pages)

59. K. Ramesh, D. Tripathi, O. Anwar Bég, A. Kadir, 'Slip and Hall current effects on viscoelastic fluid-particle suspension flow in a peristaltic hydromagnetic blood micropump', Iranian Journal of Science and Technology, Transactions of Mechanical Engineering (2018). doi.org/10.1007/s40997-018-0230-5 (18 pages)

60. M. M. Bhatti, A. Shahid, O. Anwar Bég and A. Kadir, 'Numerical study of radiative Maxwell viscoelastic magnetized flow from a stretching permeable sheet with the Cattaneo-Christov heat flux model', Neural Computing and Applications (2017). 12 pages DOI 10.1007/s00521-017-2933-8 (12 pages) 


\section{TABLES}

Table1. Grid independent test:

\begin{tabular}{|l|c|c|}
\hline Grid size & $\bar{C}_{f}$ values for $\operatorname{Pr}=1.0, G r=5.0$, & $\overline{N u}$ values for $\operatorname{Pr}=1.0, G r=5.0$, \\
& $\alpha_{1}=0.01, \alpha_{2}=0.1, \alpha_{3}=0.1$ & $\alpha_{1}=0.01, \alpha_{2}=0.1, \alpha_{3}=0.1$ \\
\hline $25 \times 125$ & 1.19858100 & 0.51026240 \\
\hline $50 \times 250$ & 1.35896500 & 0.50124320 \\
\hline $100 \times 500$ & 1.42048600 & 0.49410230 \\
\hline $200 \times 1000$ & 1.41308100 & 0.48411810 \\
\hline
\end{tabular}


Table2. Time independent test:

\begin{tabular}{|c|c|c|}
\hline Time-step & $\bar{C}_{f}$ values for $P r=1.0, G r=5.0$, & $\overline{N u}$ values for $\operatorname{Pr}=1.0, G r=5.0$, \\
size $(\Delta t)$ & $\alpha_{1}=0.01, \alpha_{2}=0.1, \alpha_{3}=0.1$ & $\alpha_{1}=0.01, \alpha_{2}=0.1, \alpha_{3}=0.1$ \\
\hline 0.1 & 1.50135100 & 0.49529500 \\
\hline 0.08 & 1.49907500 & 0.49526090 \\
\hline 0.05 & 1.49226000 & 0.49515940 \\
\hline 0.03 & 1.48018000 & 0.49498010 \\
\hline 0.01 & 1.42048600 & 0.49410230 \\
\hline
\end{tabular}

\section{FIGURES}




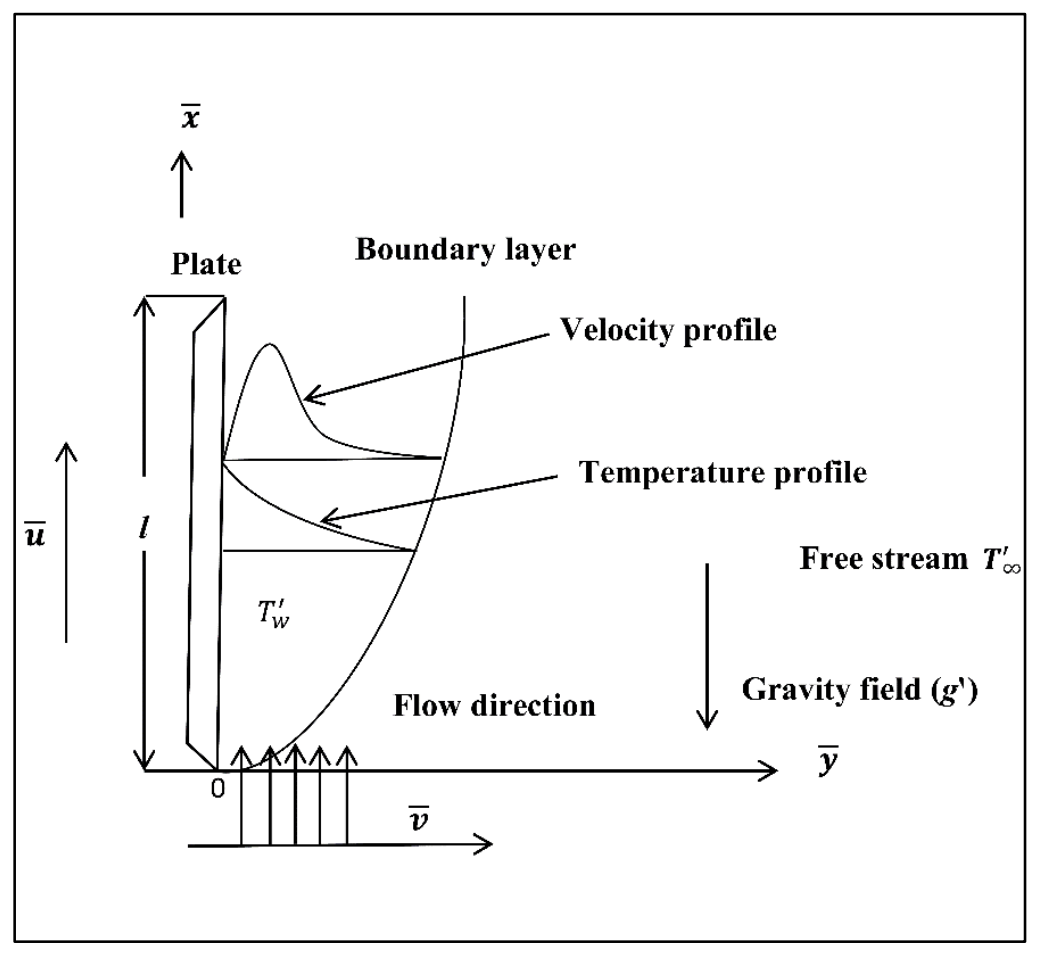

Fig. 1. Geometrical representation of current problem.

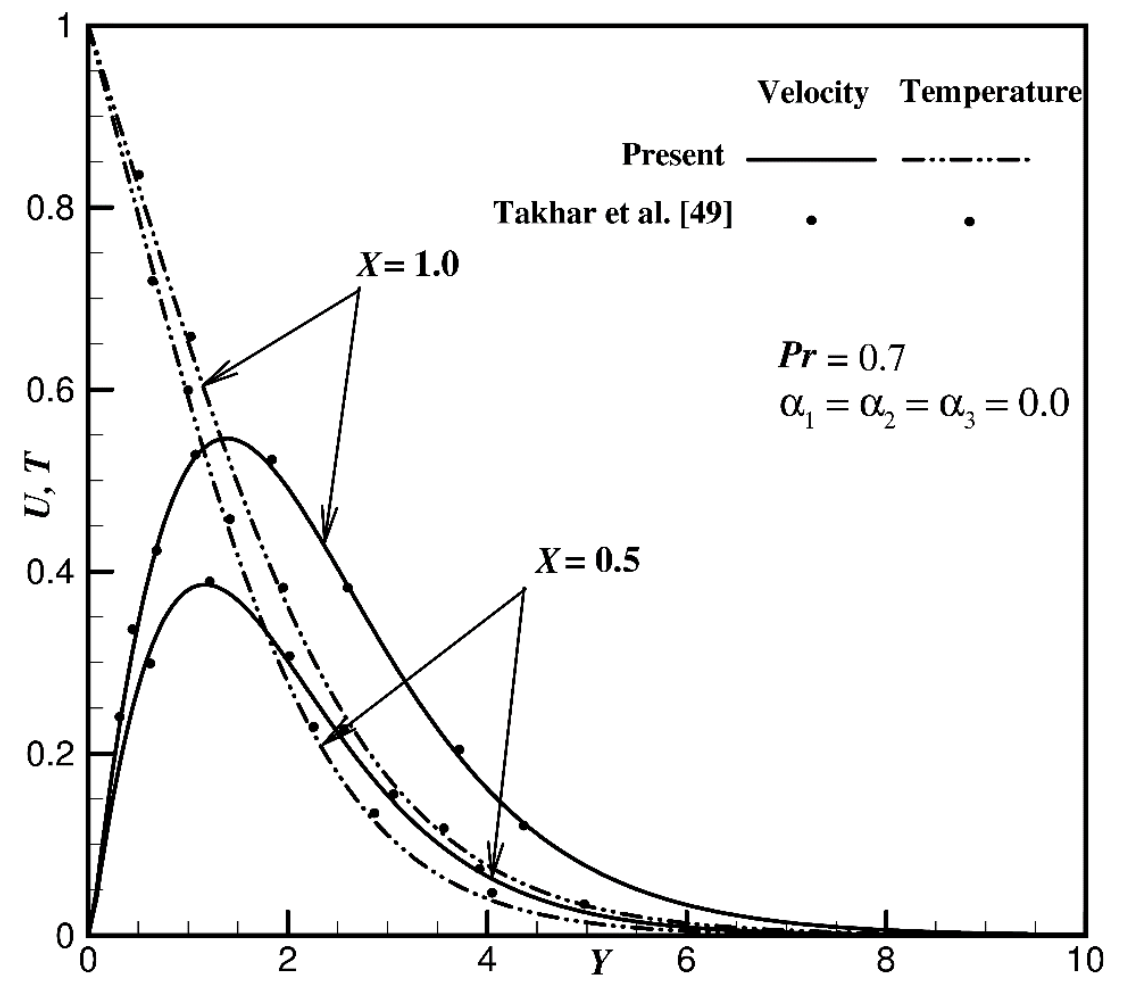


2(a)

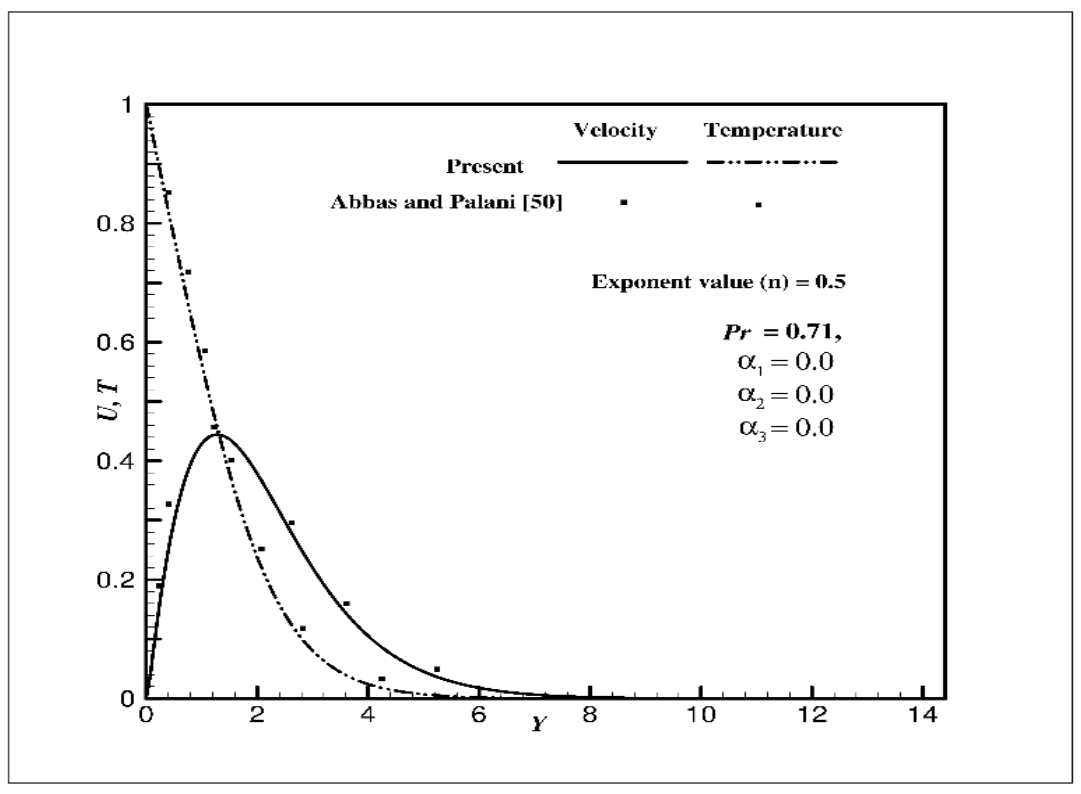

2(b)

Fig. 2. Comparison study of flow variables.

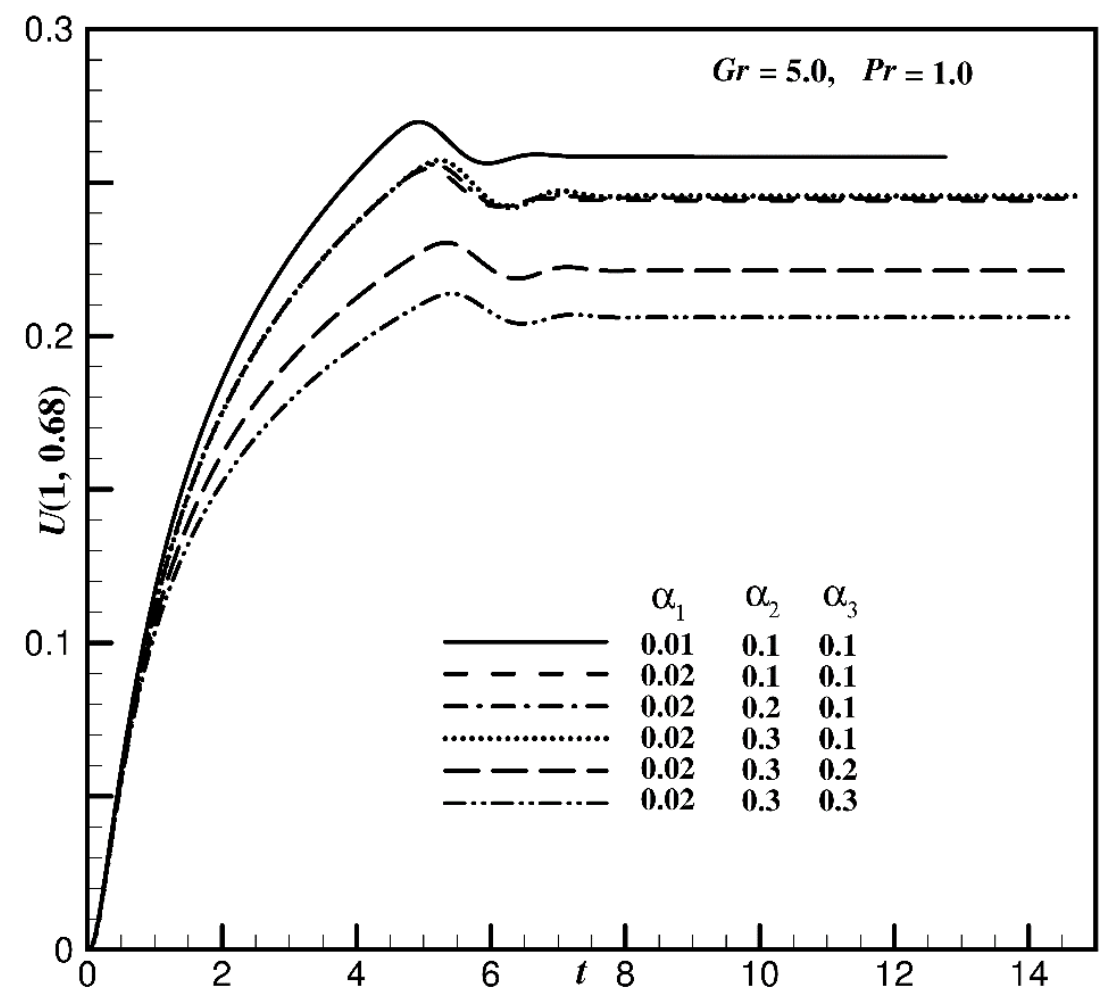

Fig. 3. Time-dependent velocity profile $(U)$ versus time $(t)$ at the point $(1,0.68)$. 


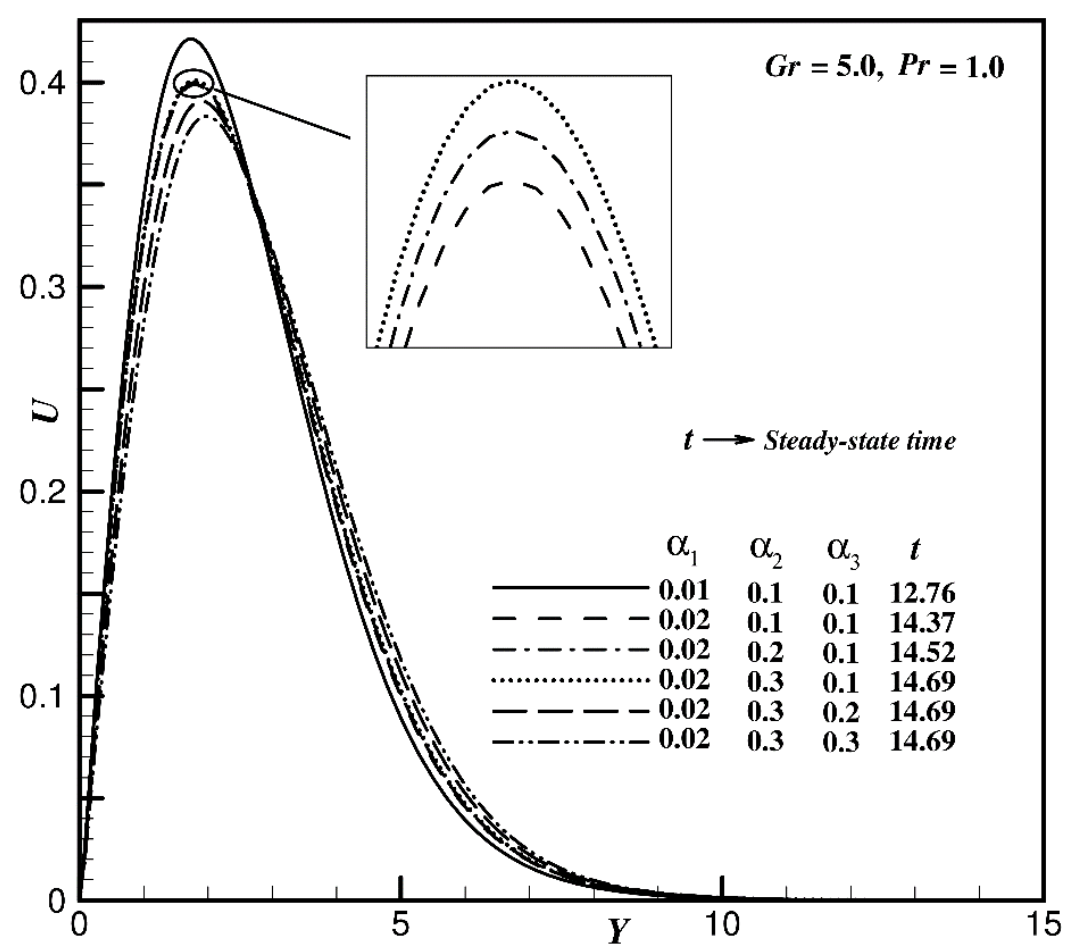

Fig. 4. Simulated time-independent state velocity profile $(U)$ versus $Y$ at $X=1.0$.

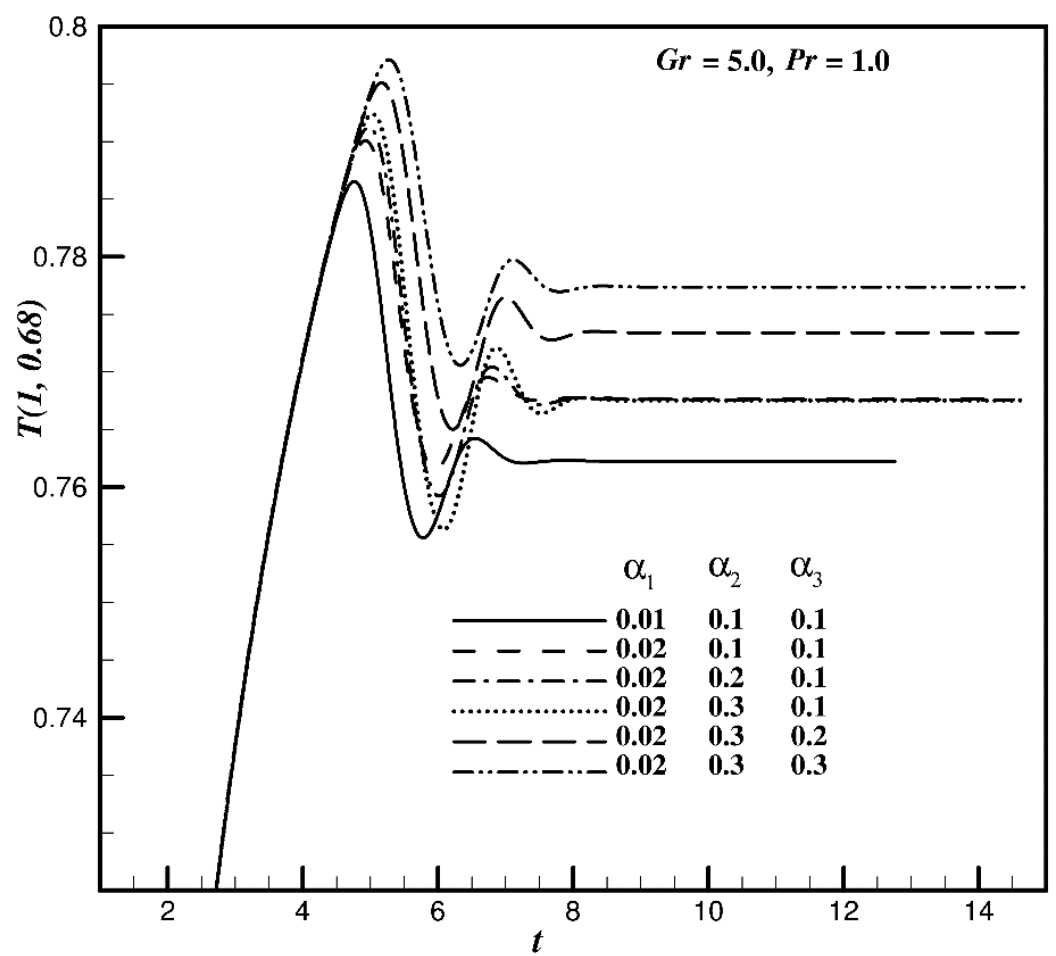

Fig. 5. Simulated time-dependent temperature profile $(T)$ versus time $(t)$ at the point $(1,0.68)$. 


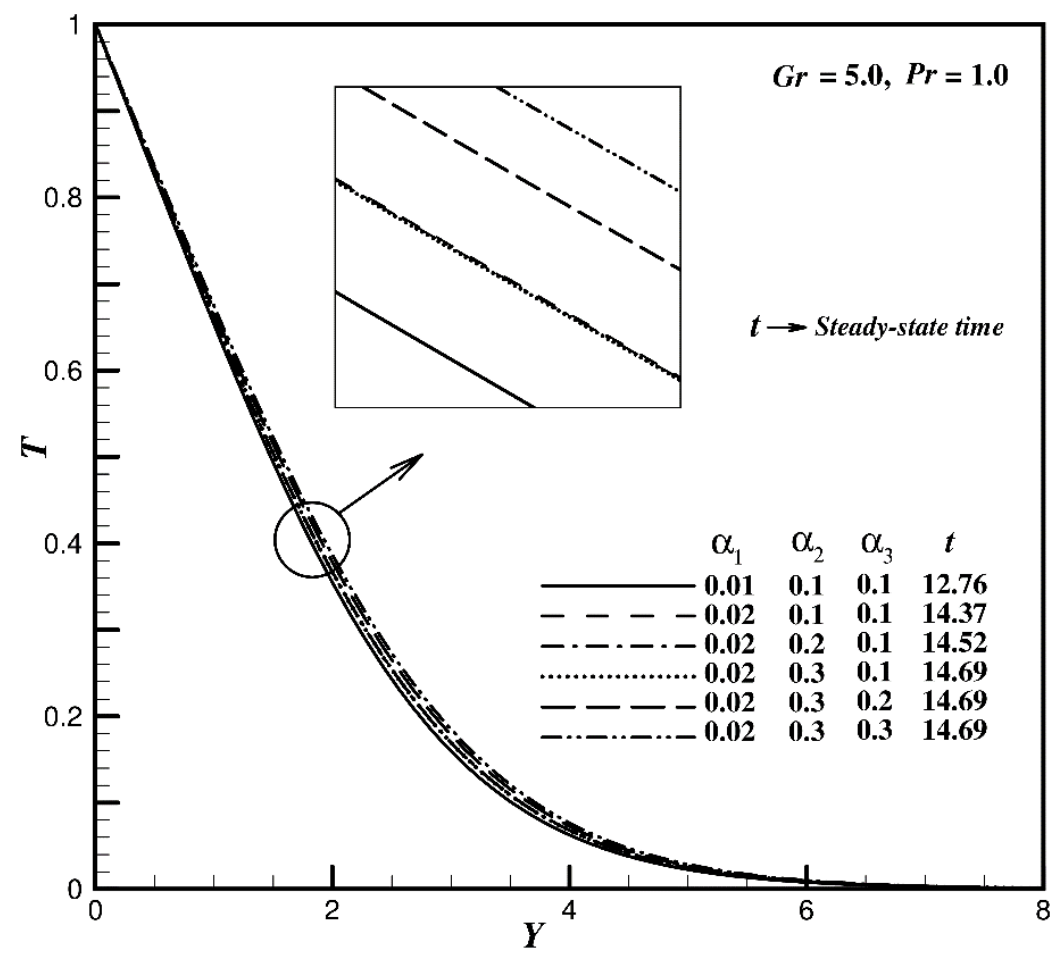

Fig. 6. Time-independent state temperature profile $(T)$ versus $Y$ at $X=1.0$.

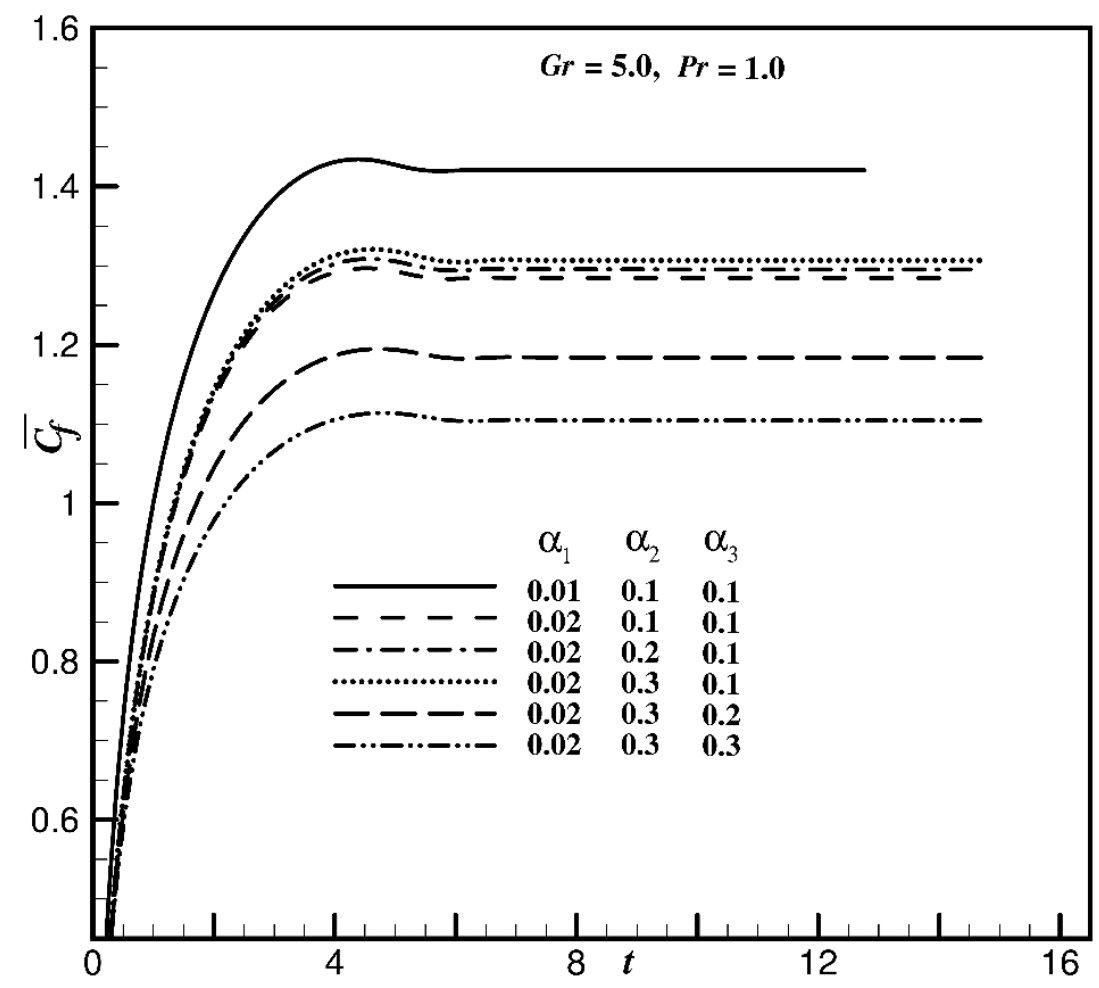

Fig. 7. Average momentum transport coefficient $\left(\overline{C_{f}}\right)$ profile against $t$. 


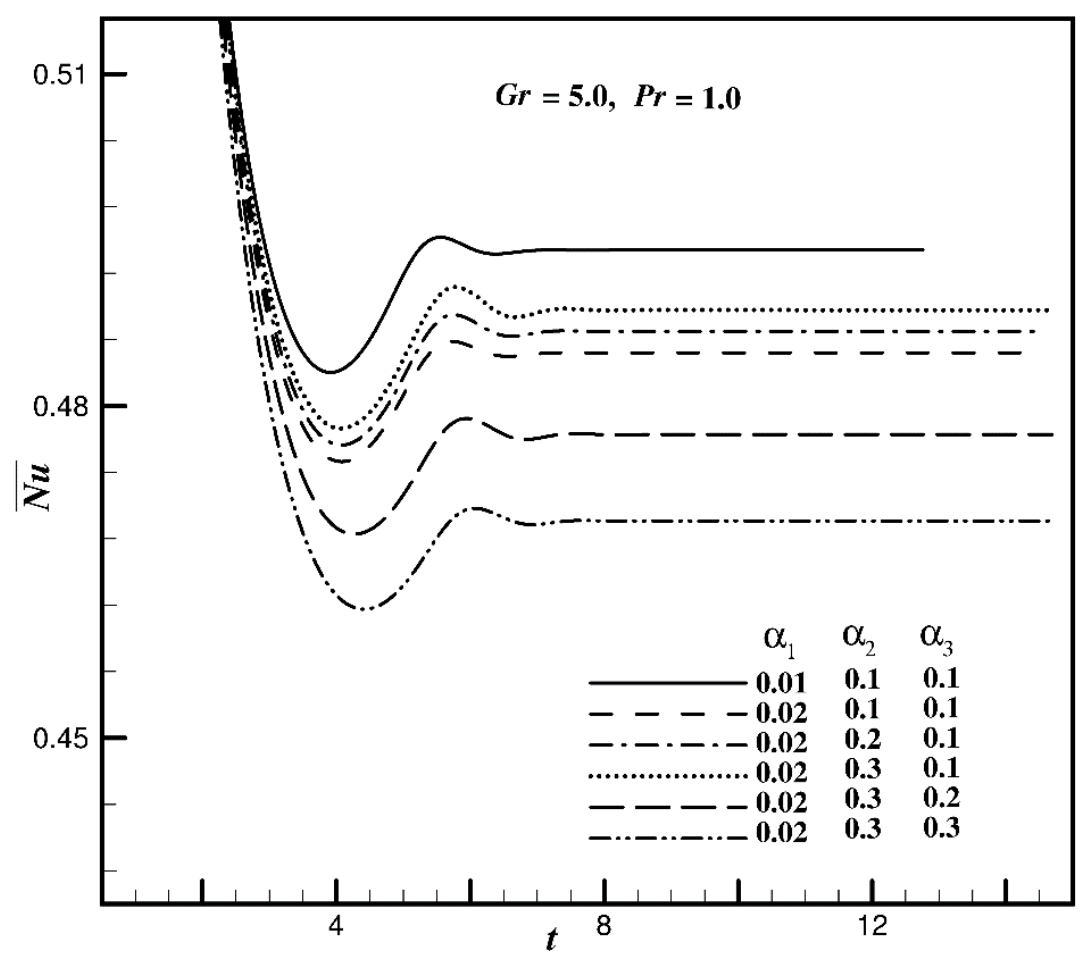

Fig. 8. Average heat transport coefficient $(\overline{N u})$ profile against $t$.

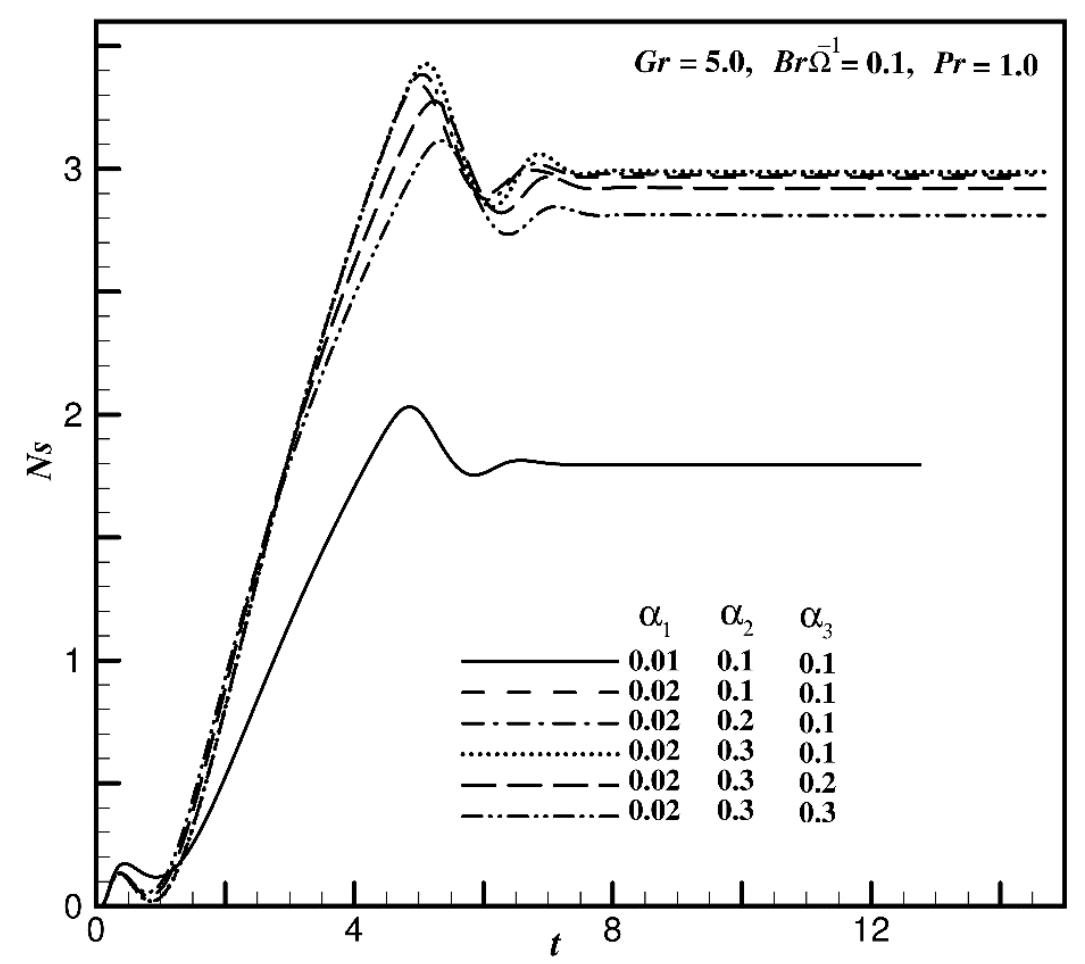

Fig. 9(a). For distinct $\alpha_{1}, \alpha_{2}$ and $\alpha_{3}$, transient graph of entropy generation (Ns). 


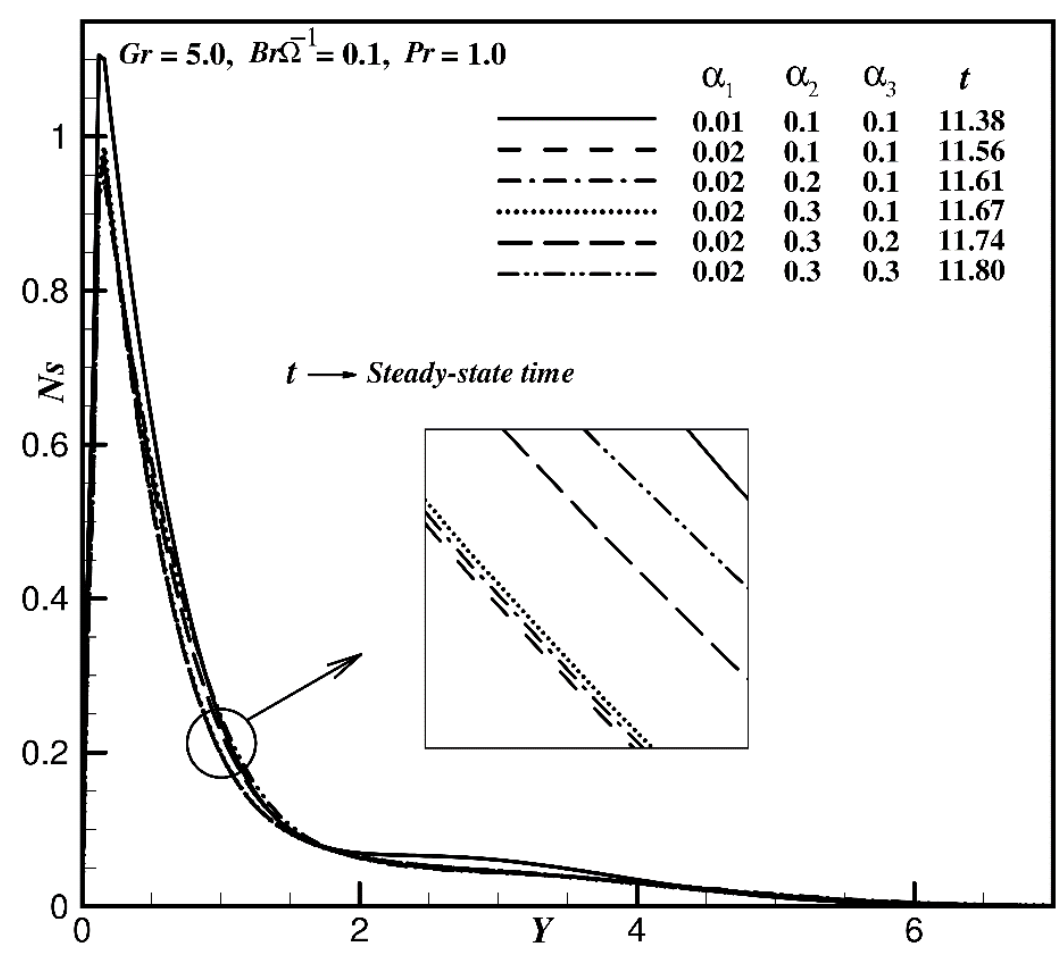

Fig. 9(b). For distinct $\alpha_{1}, \alpha_{2}$ and $\alpha_{3}$, steady-state graph of entropy generation (Ns).

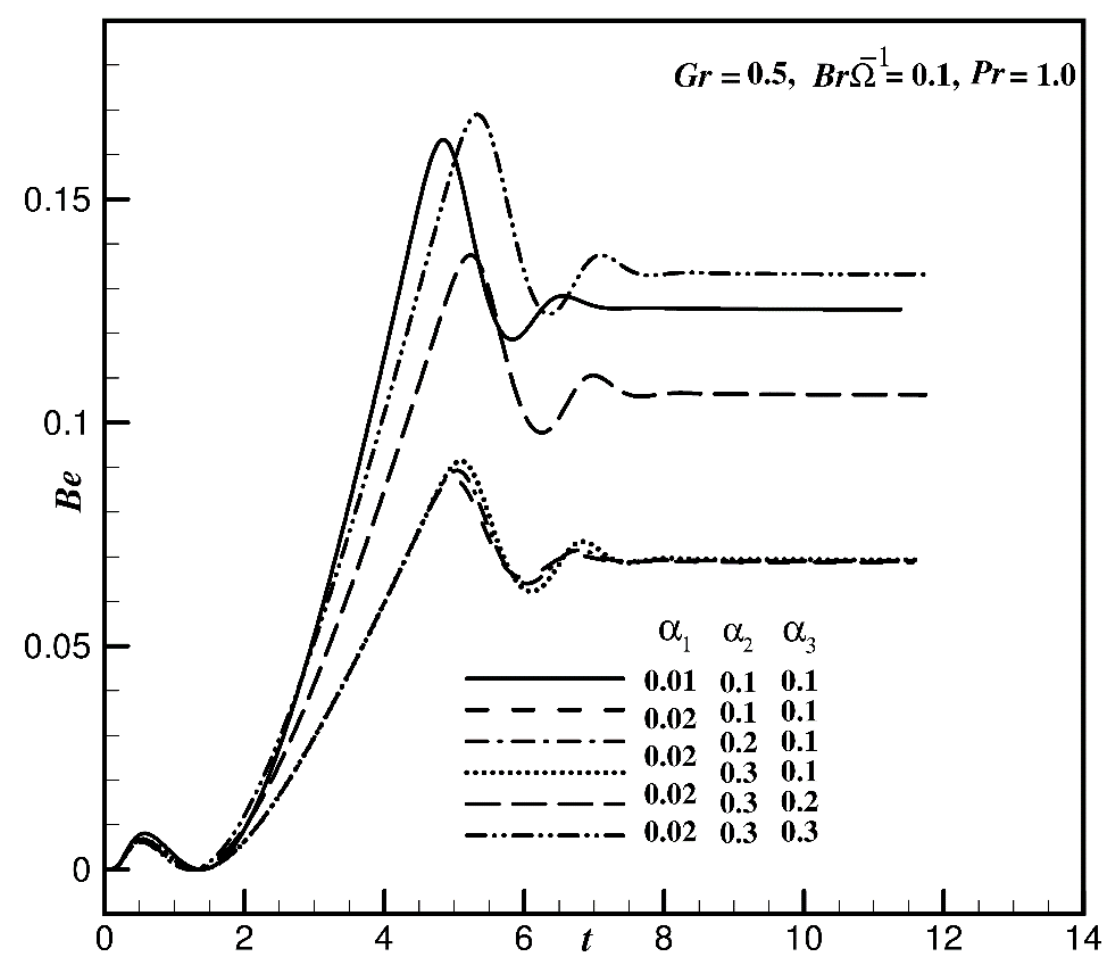

Fig. 10 (a). For different $\alpha_{1}, \alpha_{2}$ and $\alpha_{3}$, transient graph of Bejan number $(B e)$. 


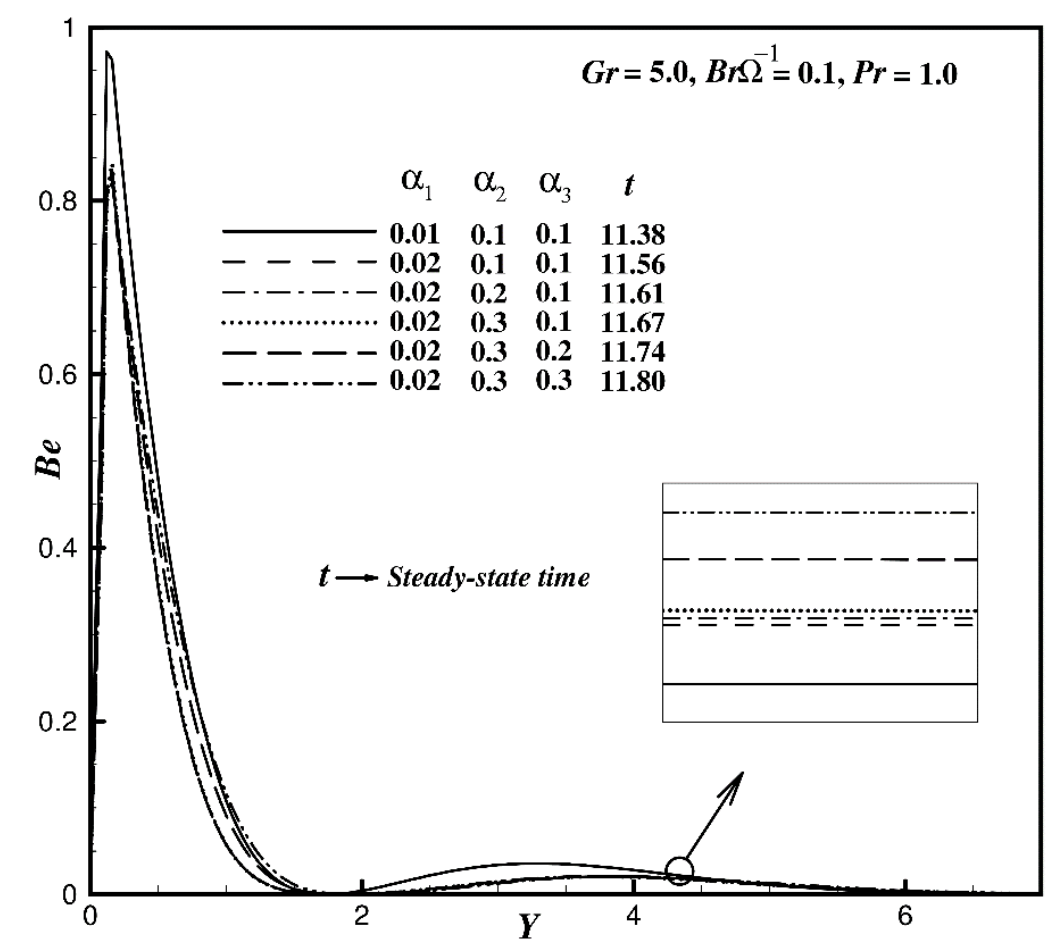

Fig. 10 (b). For different $\alpha_{1}, \alpha_{2}$ and $\alpha_{3}$, steady-state graph of Bejan number $(B e)$.

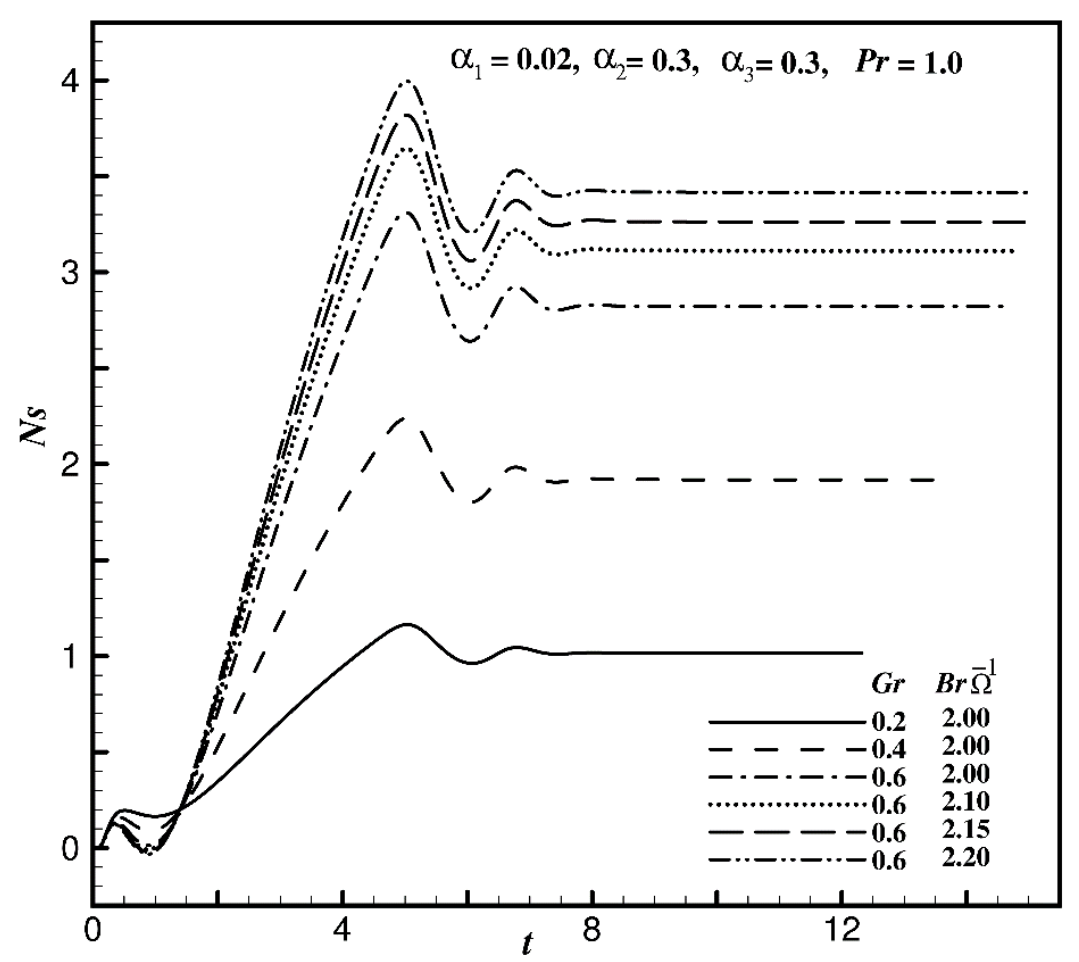

Fig. 11 (a). Simulated transient entropy profile (Ns) versus $t$ for the effect of $G r$ and $\Omega^{-1}$. 


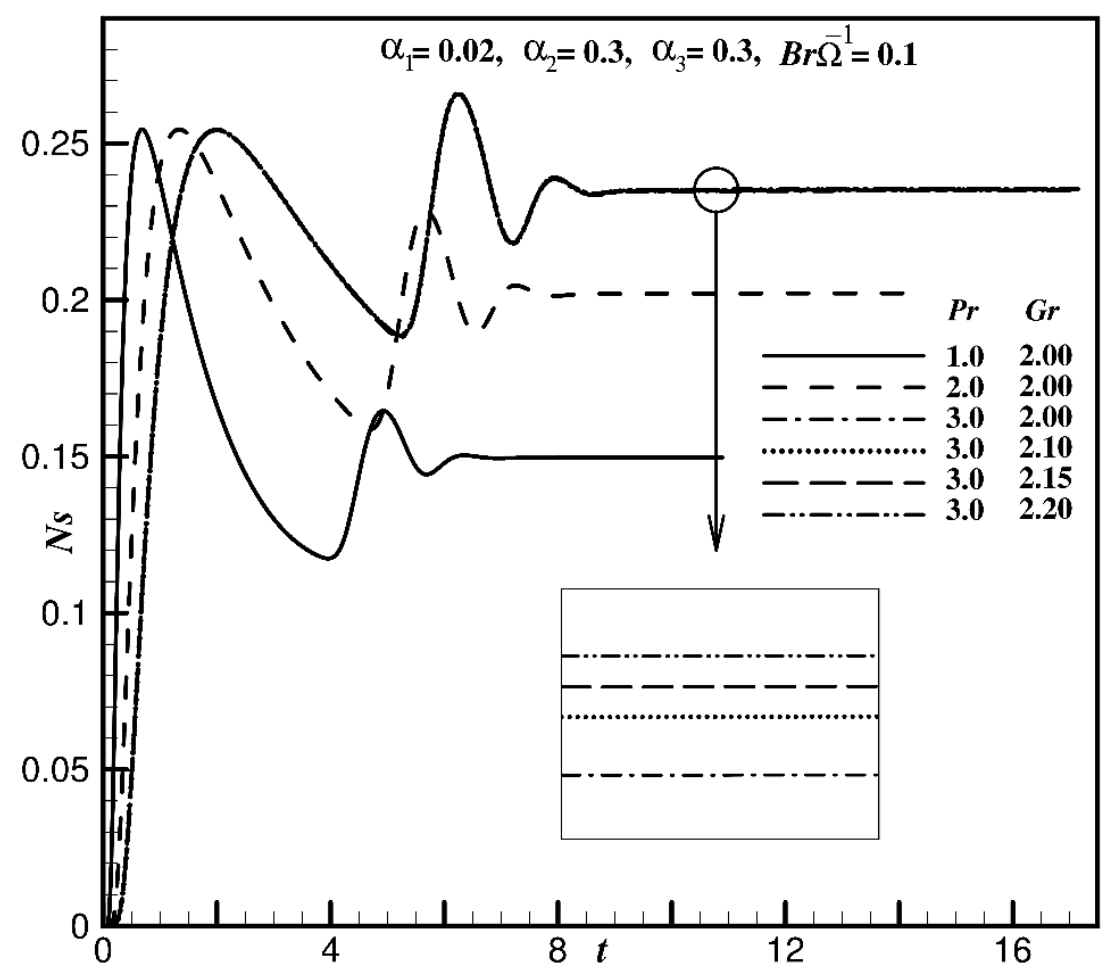

Fig. 11 (b). Simulated transient entropy profile $(N s)$ versus $t$ for the effect of $P r$ and $G r$.

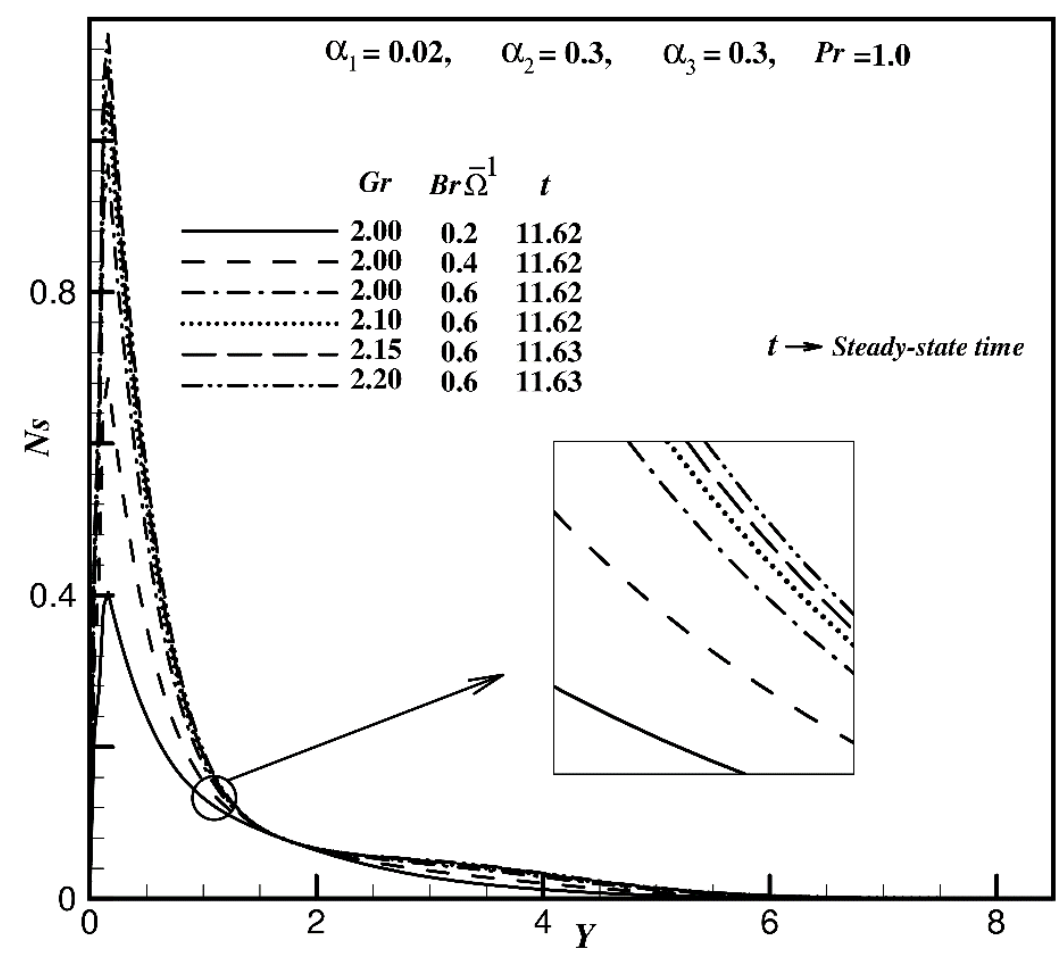

Fig. 12 (a). Steady-state entropy profile (Ns) versus $Y$ at $\mathrm{X}=1.0$ for the effect of $G r$ and $\Omega^{-1}$. 


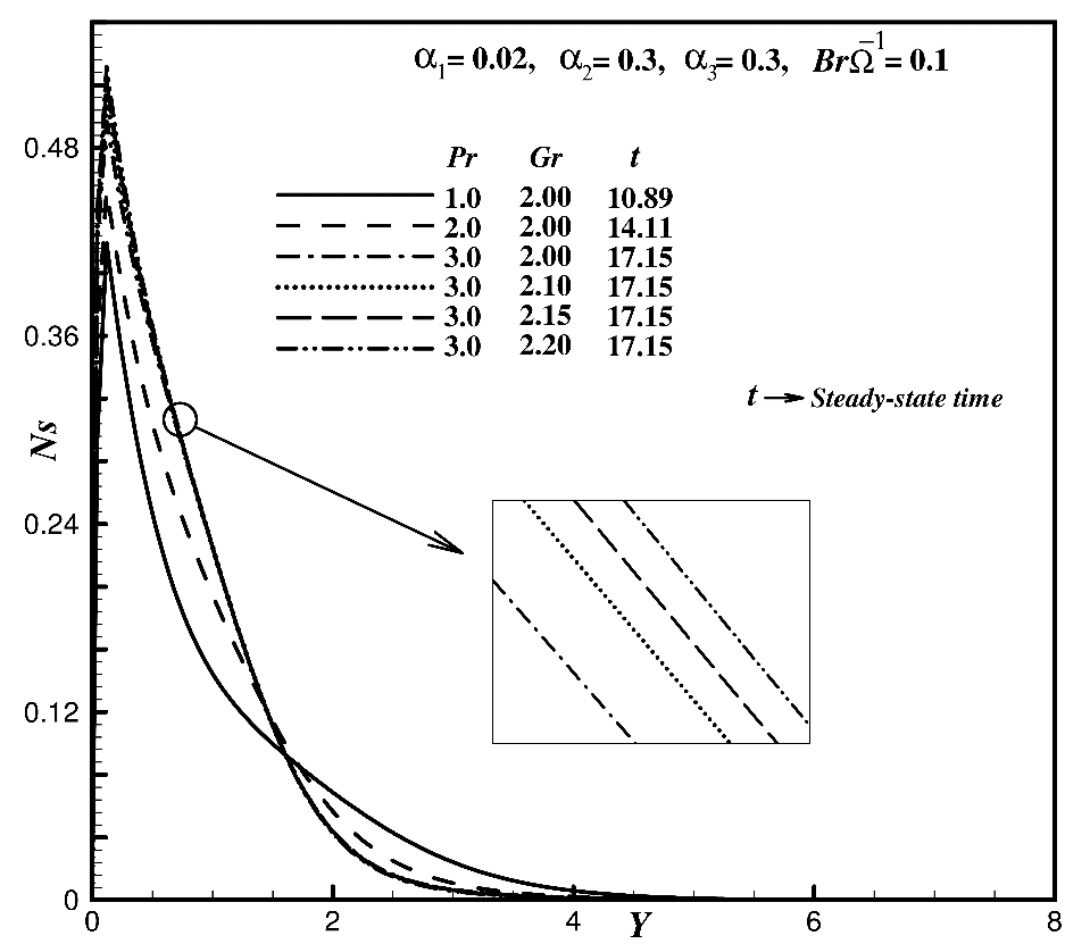

Fig. 12(b). Steady-state entropy profile $(N s)$ versus $Y$ at $\mathrm{X}=1.0$ for the effect of $P r$ and $G r$.

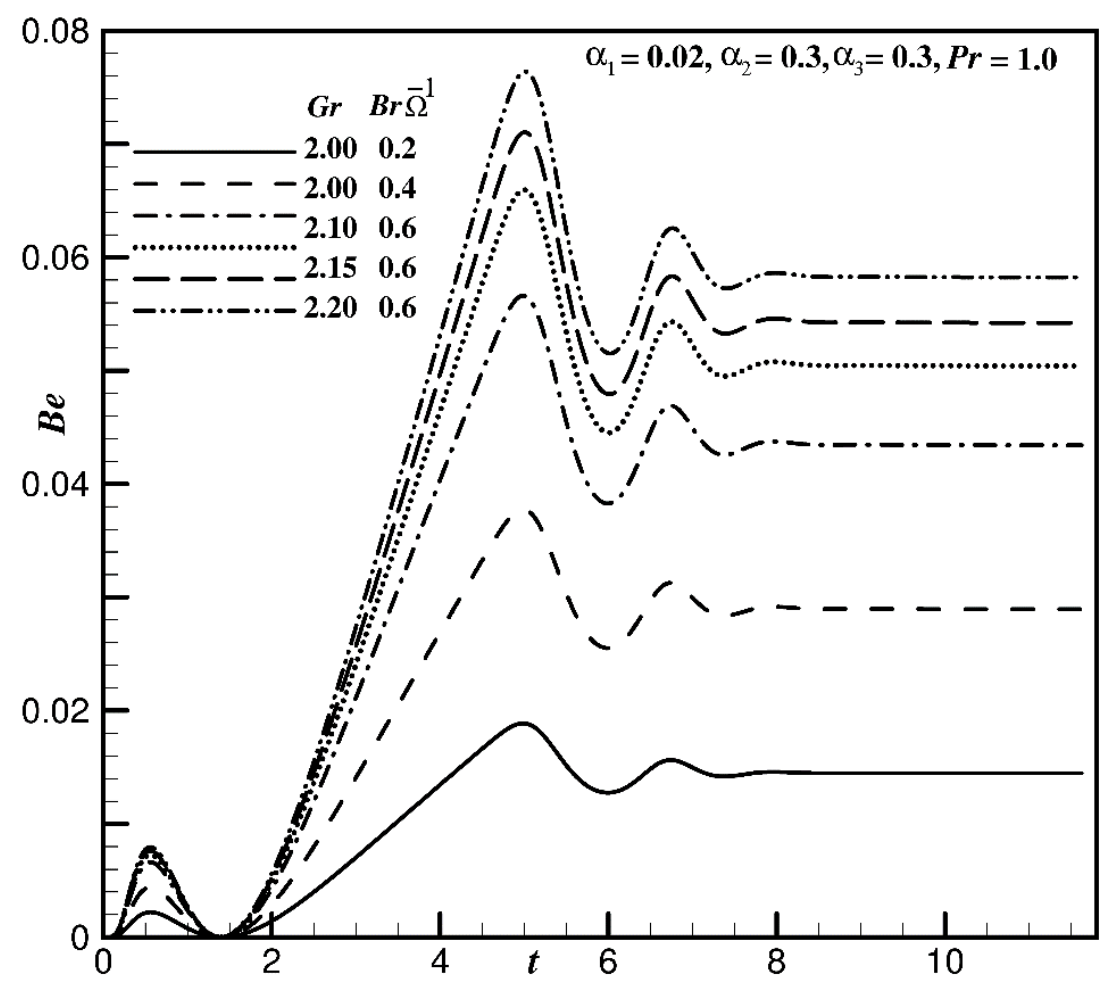

Fig. 13 (a). Simulated transient Bejan number $(B e)$ versus $t$ for the effect of $G r$ and $B r \Omega^{-1}$; 


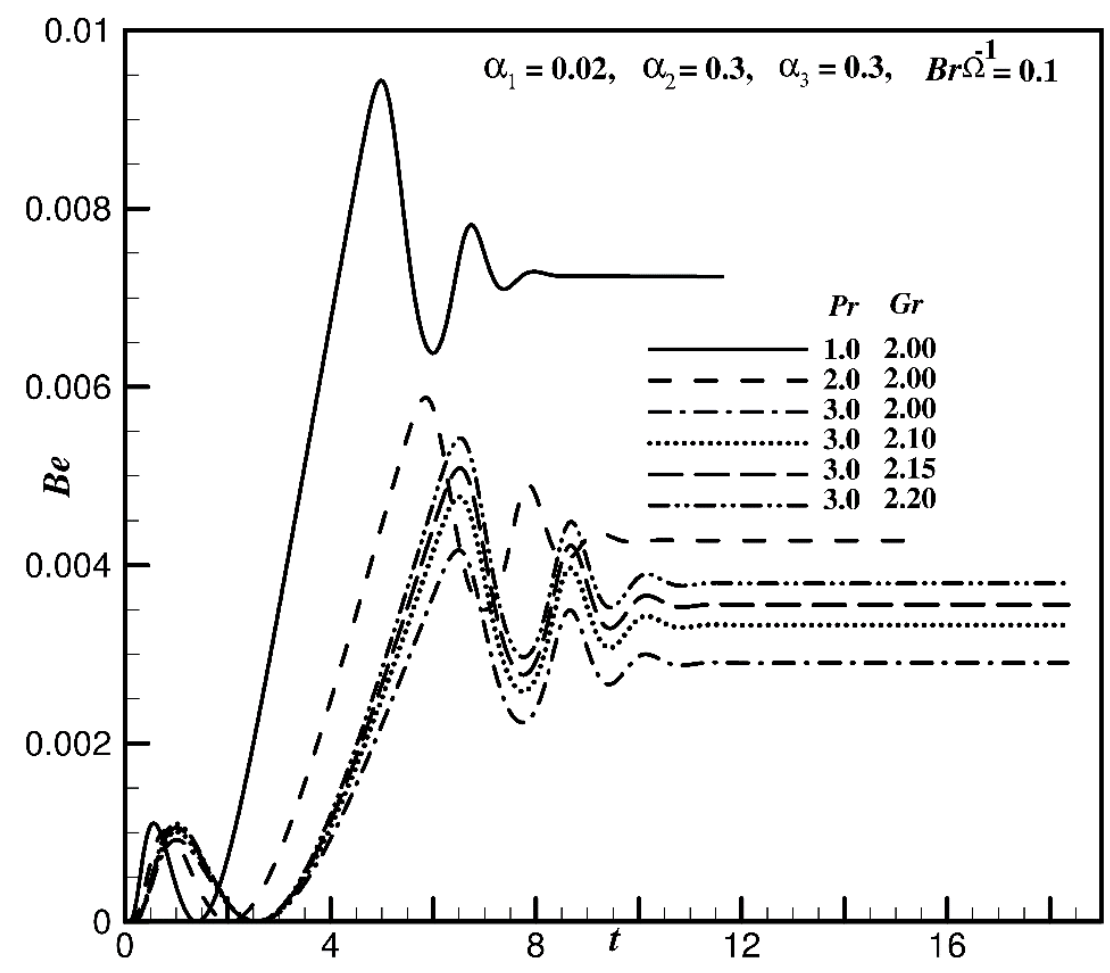

Fig. 13 (b). Simulated transient Bejan number $(B e)$ versus $t$ for the effect of $P r$ and $G r$.

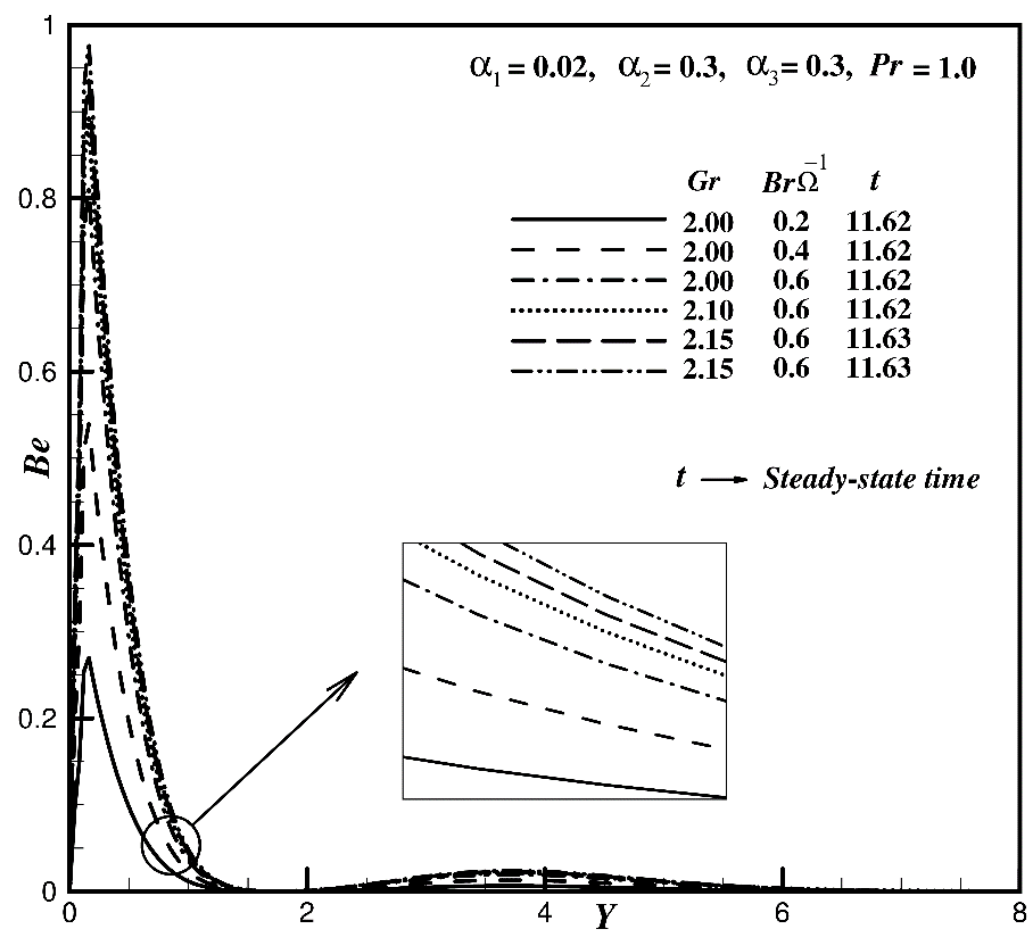

Fig. 14 (a). Time-independent Bejan number versus $Y$ at $X=1.0$ for the effect of $G r$ and $B r \Omega^{-1}$. 


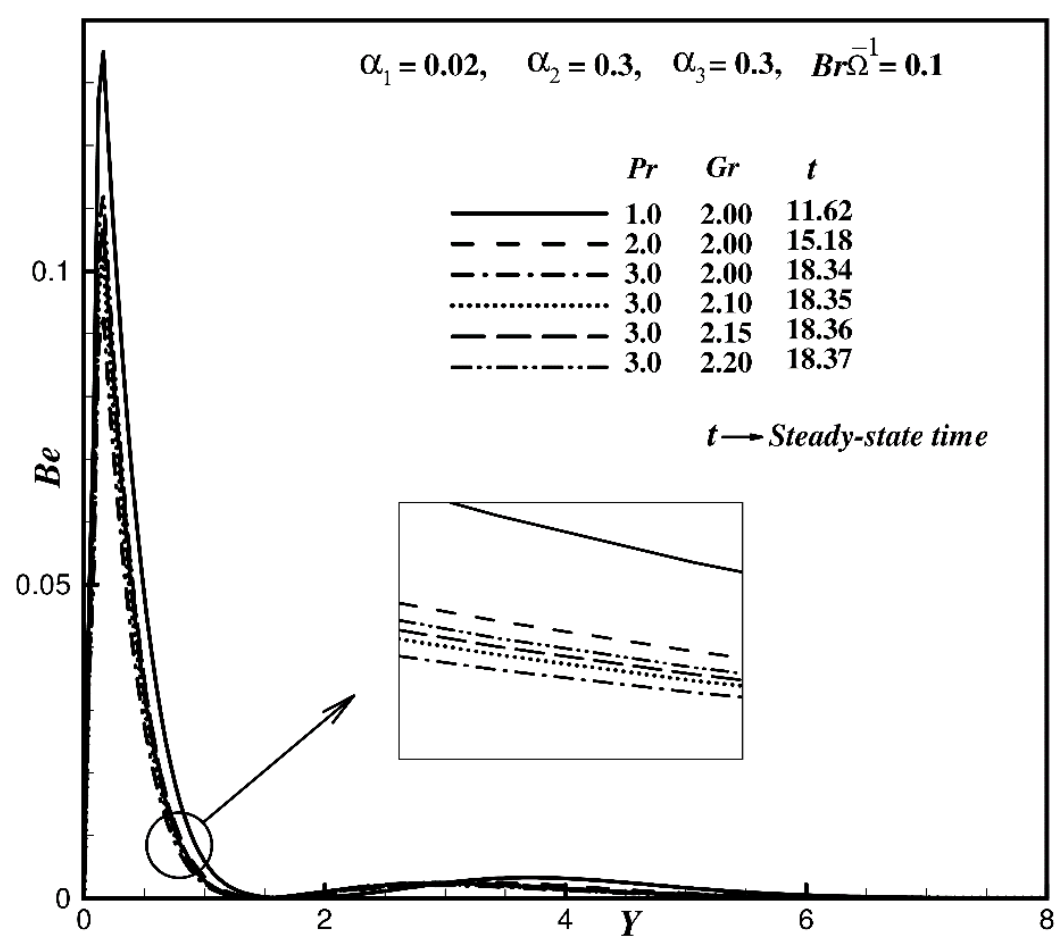

Fig. 14 (b). Time-independent Bejan number $(B e)$ versus $Y$ at $X=1.0$ for the effect of $P r$ and $G r$.
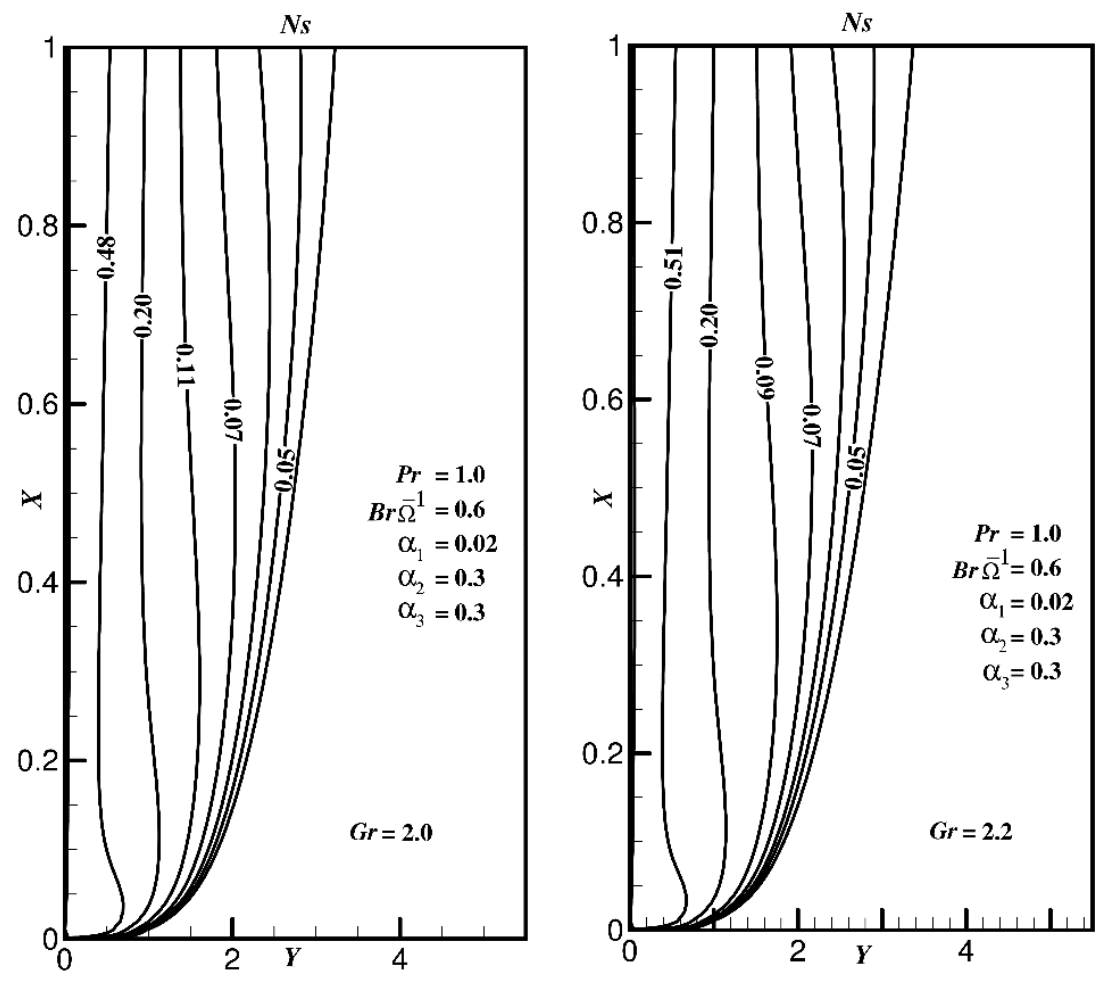

15(a) 

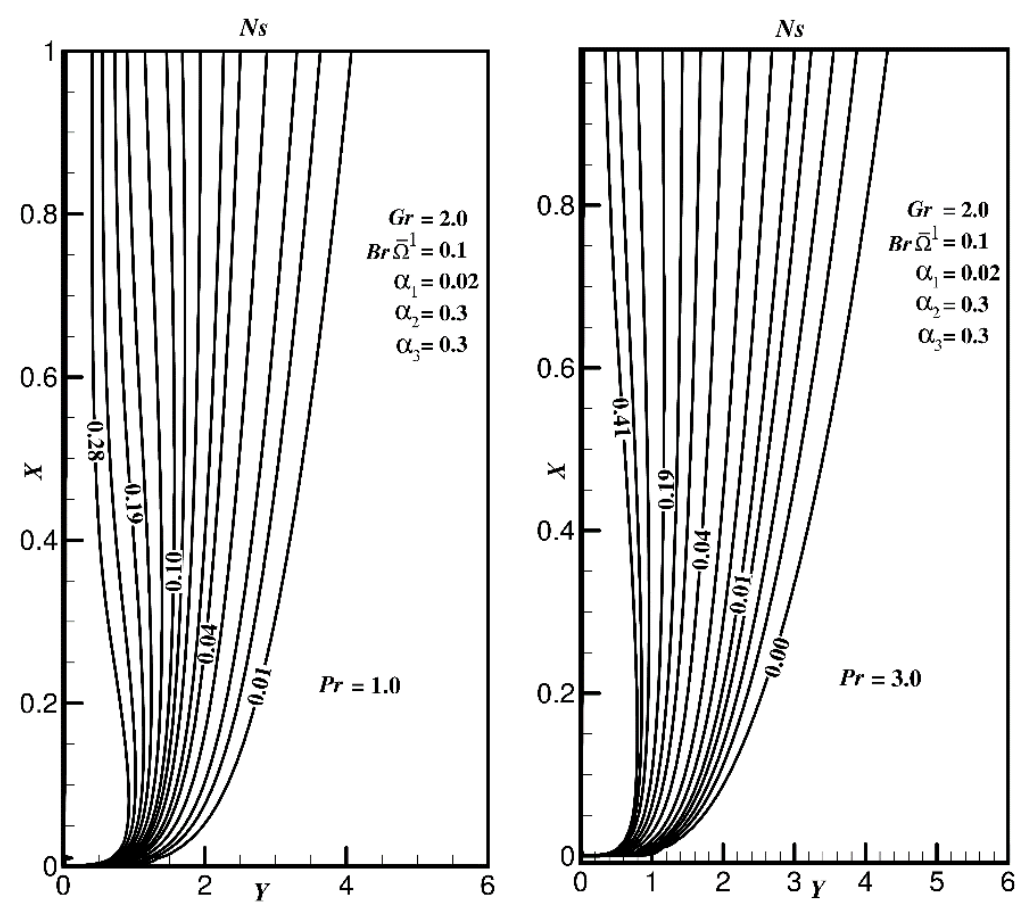

15(b)
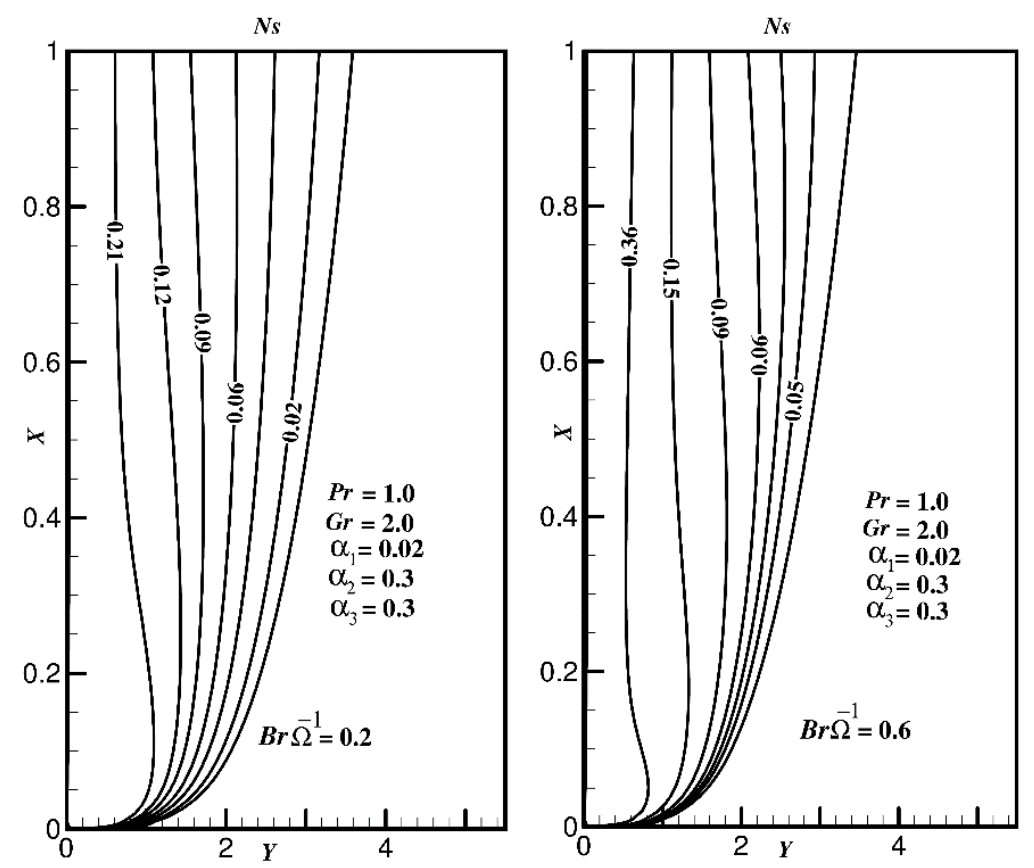

15(c) 

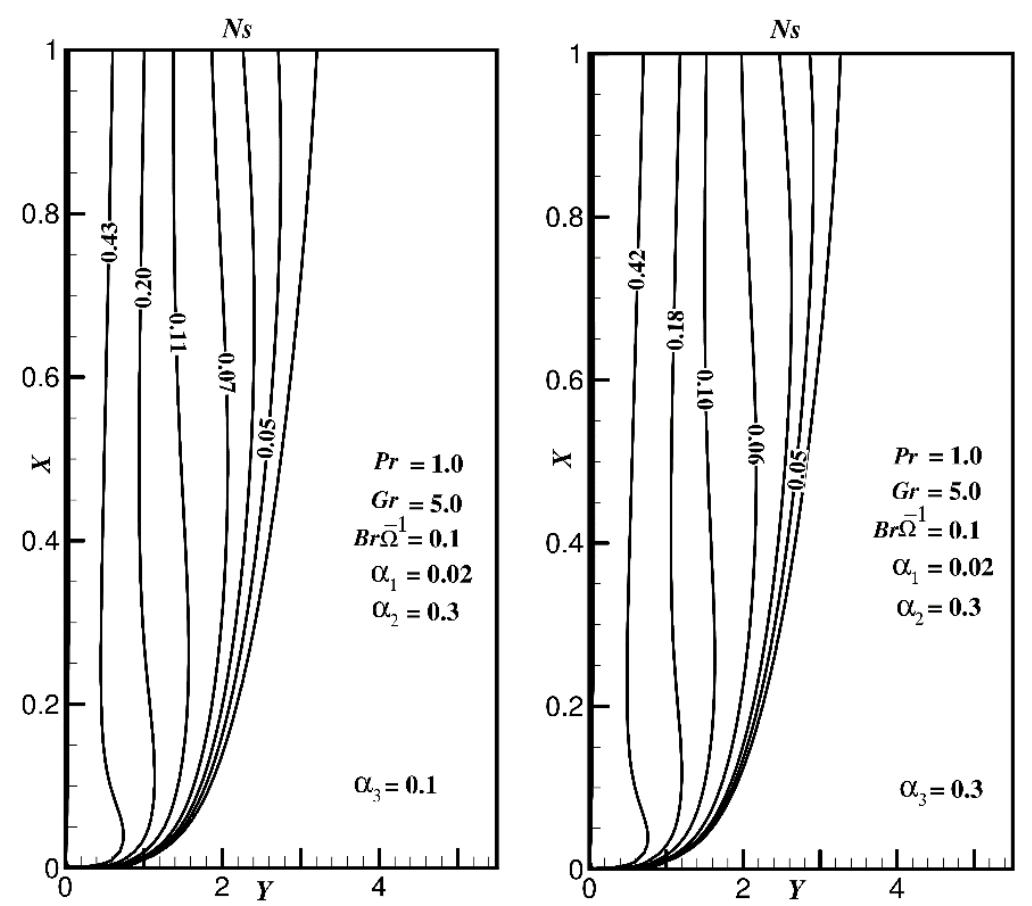

15(d)

Fig. 15. Simulated steady-state entropy lines (Ns) for different values of (a) $G r$; (b) $\operatorname{Pr}$; (c) $B r \Omega^{-1} ;$ (d) Third-grade parameter $\alpha_{3}$.
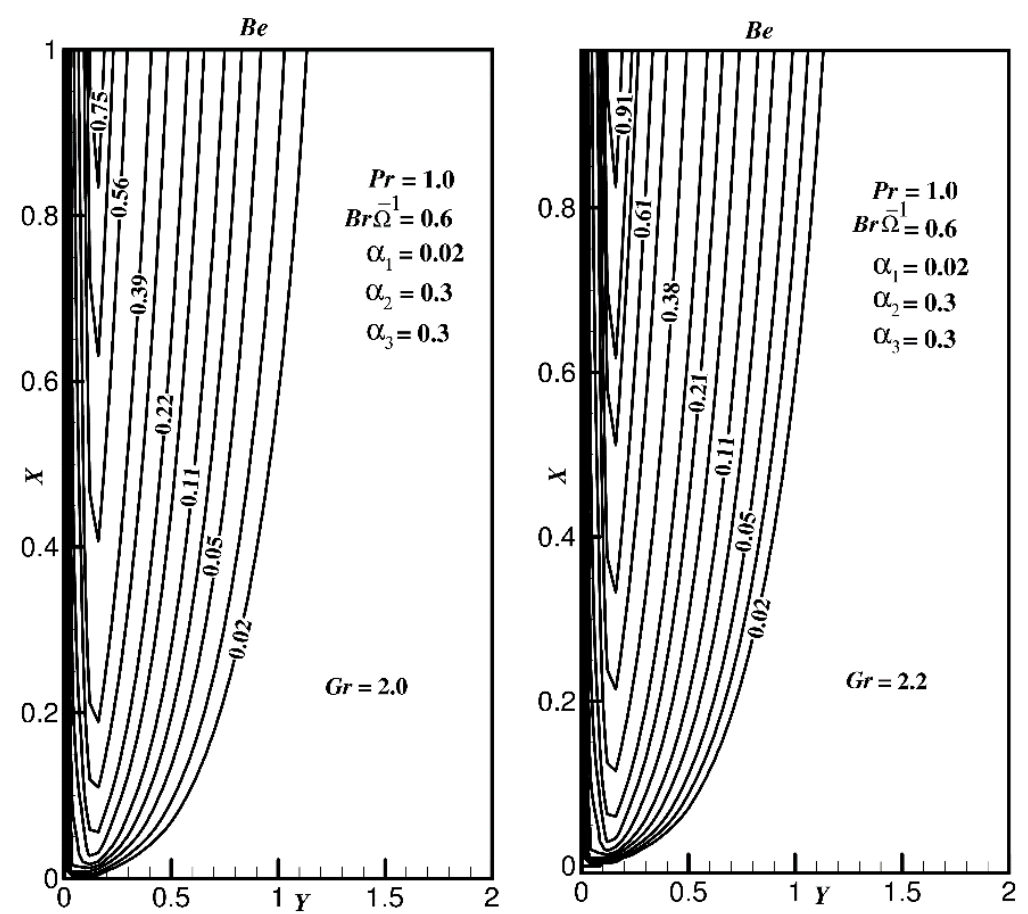
16(a)
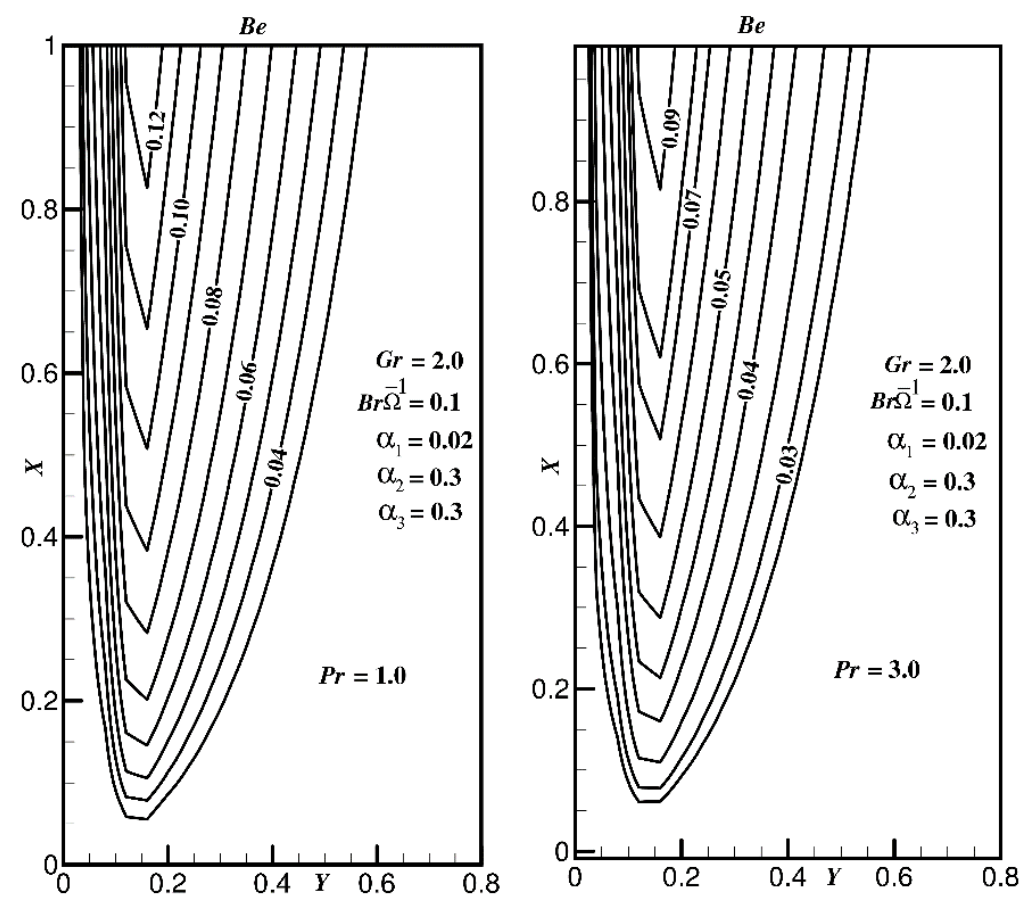

16(b)
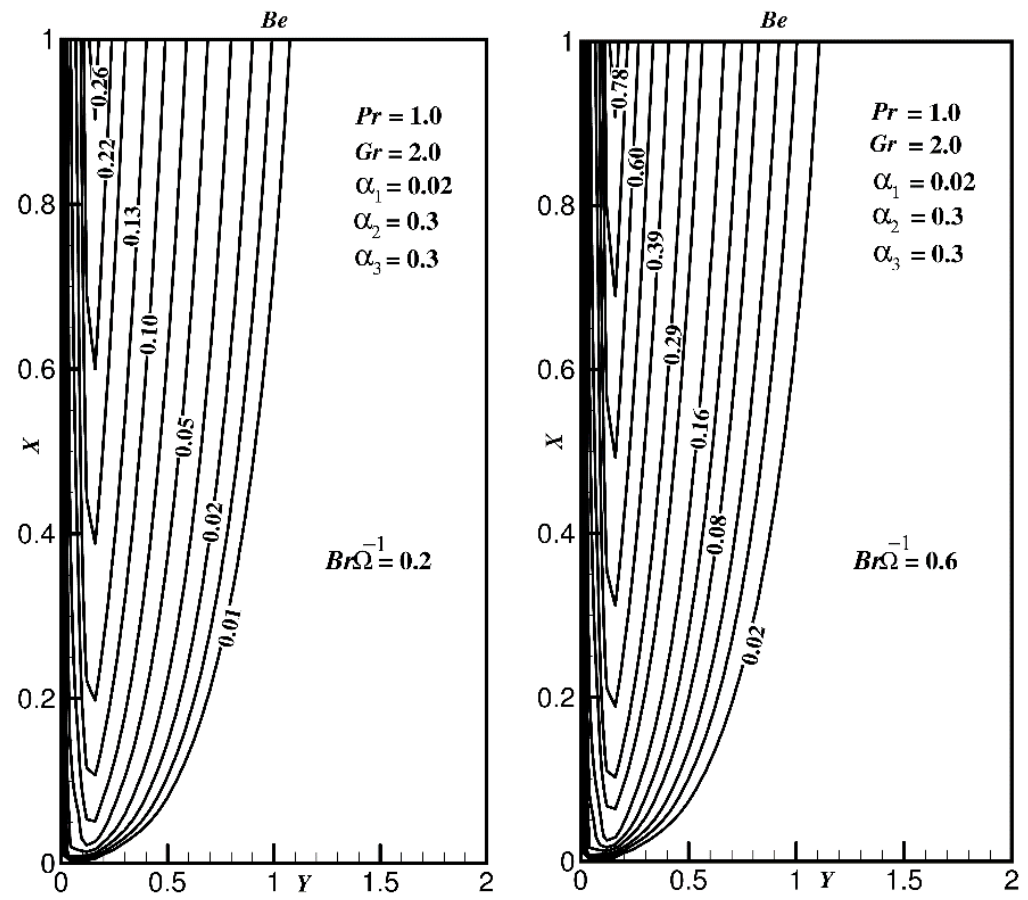

16(c) 

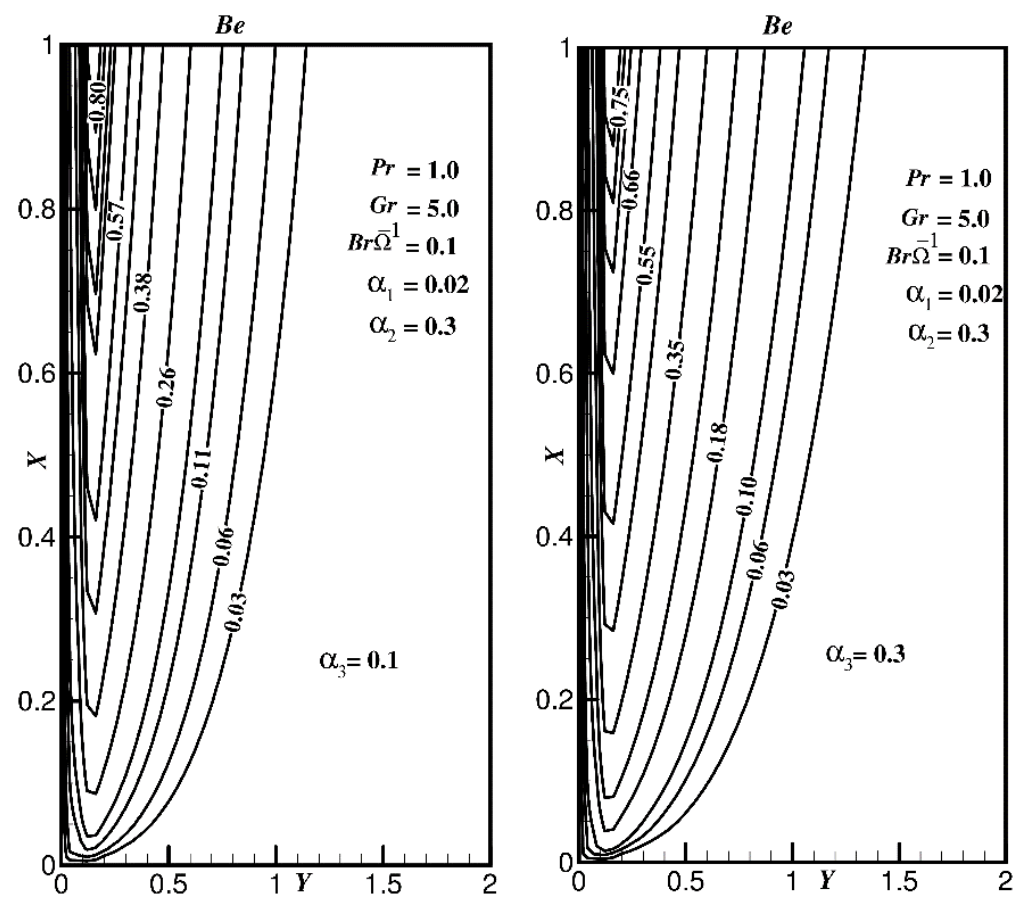

16(d)

Fig. 16. Simulated steady-state Bejan lines (Be) for different values of (a) $G r$; (b) $\operatorname{Pr}$; (c) $B r \Omega^{-1}$ ; (d) Third-grade parameter $\alpha_{3}$. 
(a)

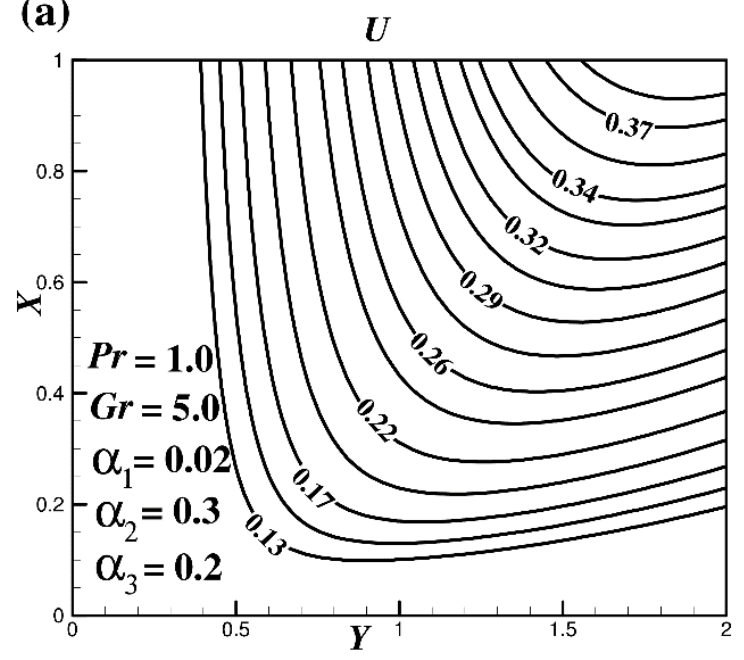

(b)

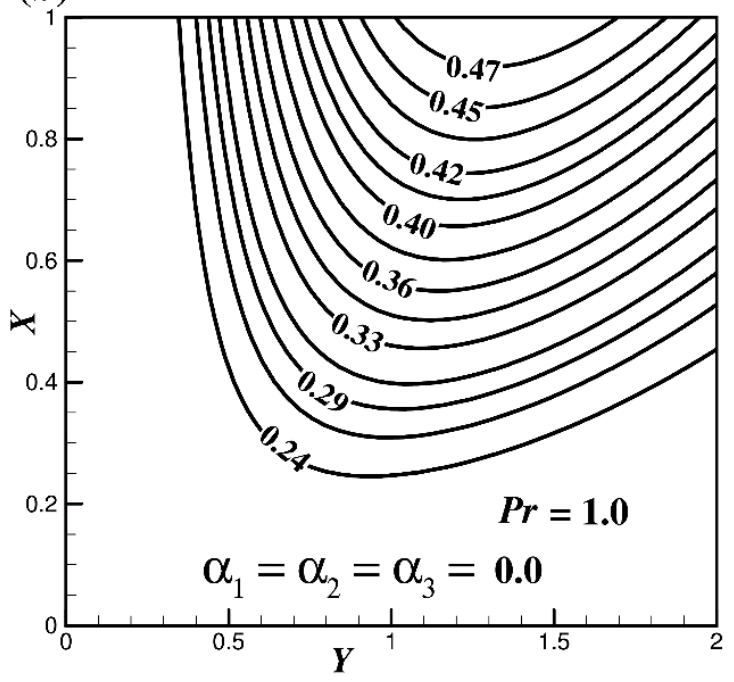

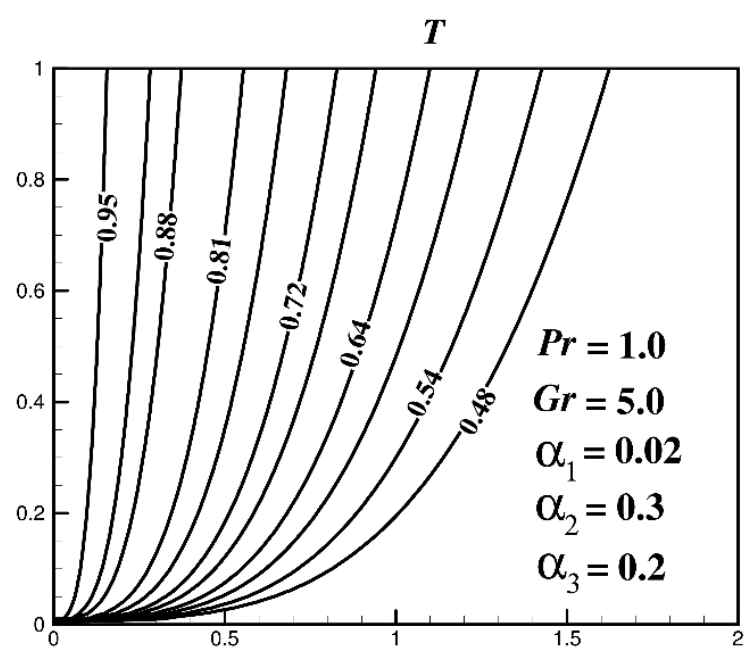

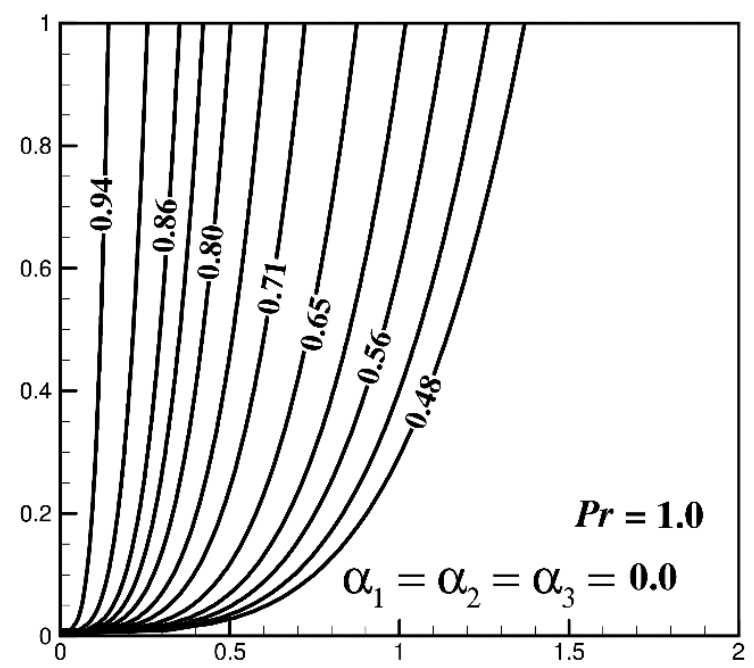

Fig. 17: Time-independent state contours of velocity $(U)$ and temperature $(T)$ for (a) Third-grade fluid ( $\alpha_{1}=0.02, \alpha_{2}=0.3, \alpha_{3}=0.2$, ); (b) Newtonian fluid $\left(\alpha_{1}=\alpha_{2}=\alpha_{3}=0.0\right)$. 